\title{
A MULTIPLICATIVE SCHWARZ ADAPTIVE WAVELET METHOD FOR ELLIPTIC BOUNDARY VALUE PROBLEMS
}

\author{
ROB STEVENSON AND MANUEL WERNER
}

\begin{abstract}
A multiplicative Schwarz overlapping domain decomposition method is considered for solving elliptic boundary value problems. By equipping the relevant Sobolev spaces on the subdomains with wavelet bases, adaptive wavelet methods are used for approximately solving the subdomain problems. The union of the wavelet bases forms a frame for the Sobolev space on the domain as a whole. The resulting method is proven to be optimal in the sense that, in linear complexity, the iterands converge with the same rate as the sequence over $N \in \mathbb{N}$ of the best approximation from the span of the best $N$ frame elements. Numerical results are given for the method applied to Poisson's equation.
\end{abstract}

\section{INTRODUCTION}

In CDD01, CDD02 by Cohen, Dahmen and DeVore, adaptive wavelet methods were developed for solving well-posed linear operator equations, such as elliptic boundary value problems in variational form. Starting with some wavelet basis for the (Sobolev) space that contains the solution, these methods were shown to be (quasi-) optimal in the sense that, in linear complexity, they converge in the energy norm with the best possible rate, i.e., the rate of the sequence over $N \in \mathbb{N}$ of the best approximation to the solution from the span of the best $N$ wavelets from the basis.

Except for rectangular domains, or smooth images of such, a bottleneck for the application of these methods is the construction of a suitable wavelet basis on the domain $\Omega$ on which the equation is posed. Starting from a nonoverlapping decomposition of $\Omega$ into subdomains, each being a smooth parametric image of the unit cube, various construction principles have been developed. Unfortunately, the resulting bases either do not (fully) satisfy the conditions needed for proving optimality (e.g., wavelets supported near interfaces between subdomains do not have enough vanishing moments or are not sufficiently smooth), or their condition numbers are very large. This was the motivation to study adaptive wavelet frame methods in Ste03, DFR07, $\mathrm{DFR}^{+}$07].

Based on an overlapping decomposition of $\Omega$ into subdomains $\left\{\Omega_{i}\right\}$, a wavelet frame is simply obtained by taking the union of wavelet bases on the subdomains. In frame coordinates, the operator equation under consideration now corresponds to a singular, but consistent bi-infinite matrix-vector equation. This equation can

Received by the editor March 6, 2008 .

2000 Mathematics Subject Classification. Primary 65N55, 65T60, 41A25.

Key words and phrases. Elliptic boundary value problems, wavelets, frames, adaptivity, best $N$-term approximation, multiplicative Schwarz method, domain decomposition.

(C)2008 American Mathematical Society 
be solved by applying an inexact Richardson (cf. [Ste03]), or steepest descent iteration (see [DFR ${ }^{+}$07]), similar in spirit to the wavelet basis method from [CDD02]. Under some technical condition on the frame, these methods were shown to be quasi-optimal in the aforementioned sense. Unfortunately, so far this technical condition, although reasonable, could be rigorously verified in a model case only. More importantly, numerical results showed that, in a quantitative sense, one has to pay for solving a system that is singular. Errors made in inexact matrix-vector products that happen to be in the kernel of the matrix will never be damped in subsequent iterations. Although they have no influence on the resulting approximations, they have to be restricted to a sufficiently small size in order to control the cost of subsequent inexact matrix-vector products.

In this paper, a method is studied that is based on the easy wavelet frame construction whilst the inversion of singular systems is avoided. Since the Sobolev space on the domain as a whole has a stable decomposition into corresponding Sobolev spaces on the subdomains subject to appropriate homogeneous Dirichlet boundary conditions, the continuous problem can be solved by the multiplicative or properly damped additive Schwarz method. For the recurrent approximate solving of the subdomain problems, an adaptive wavelet basis method is applied.

Although the latter method is (quasi-) optimal, it is not clear that this will hold for the overall method. Indeed, suppose that the solution $u$ of the boundary value problem can be approximated by linear combinations of $N$ wavelet frame elements such that the error is $\mathcal{O}\left(N^{-s}\right)$, i.e., that the rate of best $N$-term approximation is $s$. For a range of $s$, depending on the order of the equation and on that of the wavelets, we will show that this holds true if and only if $u$ is in a certain smoothness space $\mathcal{B}^{s}(\Omega)$, being very close (but not equal to) some Besov space. On each of the subdomains, the iterands will converge to some $u^{(i)}$, necessarily with $\sum_{i} u^{(i)}=u$. With a standard implementation of the Schwarz method, it is not clear whether this splitting is smoothness preserving in the sense that each $u^{(i)} \in \mathcal{B}^{s}\left(\Omega_{i}\right)$, being a necessary, and by optimality of the adaptive wavelet basis method, sufficient condition for the iterands on the subdomain to converge with rate $s$.

In this paper, we restrict ourselves to the multiplicative Schwarz method. In order to deal with the above problem, before solving on subdomain $\Omega_{i}$, we will remove terms from the expansions of iterands associated to other subdomains that correspond to wavelets that are fully supported in $\Omega_{i}$. In any case with an exact solving on $\Omega_{i}$, this modification does not change the resulting next global approximation to $u$. It does, however, change the splitting $u=\sum_{i} u^{(i)}$. Assuming a sufficiently large overlap between the subdomains compared to the maximal support sizes of the wavelets, we will prove that now $\left\|u_{i}\right\|_{\mathcal{B}^{s}\left(\Omega_{i}\right)}$ can be bounded by some absolute multiple of $\|u\|_{\mathcal{B}^{s}(\Omega)}$, which will imply (quasi-) optimality of the resulting method. Since additionally our modification diminishes the transport of information between subdomains, which generally is relatively expensive due to uncorrelated underlying meshes, it results also in a more efficient implementation. In a way our approach is close to the original method proposed by Schwarz (cf. Sch1890), in which data from other subdomains are only passed as Dirichlet boundary conditions.

The remainder of this paper is organized as follows. In Section 2 we describe the boundary value problem and the (exact) multiplicative Schwarz method for solving it. Furthermore, we collect standard assumptions on the wavelets we will consider. Section 3 is devoted to the development of the adaptive multiplicative 
Schwarz method. We prove convergence and optimality of this algorithm where for the latter we use some technical results that will be shown in Sections 4 and 5 . In particular, in Section 4, we give explicit expressions for the limits $u^{(i)}$ of the sequences of approximate solutions of the subdomain problems. In Section 5, we show that the splitting of the solution $u$ of the boundary value problem into these $u^{(i)}$ is smoothness preserving. Finally, in Section 6, we present numerical results obtained with the multiplicative adaptive Schwarz method that support our theoretical findings.

In this paper, by $C \lesssim D$ we will mean that $C$ can be bounded by a multiple of $D$, independently of parameters which $C$ and $D$ may depend on. Obviously, $C \gtrsim D$ is defined as $D \lesssim C$, and $C \approx D$ as $C \lesssim D$ and $C \gtrsim D$.

\section{Preliminaries}

2.1. Boundary value problem. For some bounded domain $\Omega \subset \mathbb{R}^{n}$ and $t \in \mathbb{N}$, let $a(\cdot, \cdot)$ be a bounded, symmetric and elliptic bilinear form on $H_{0}^{t}(\Omega)$. For given $f \in H^{-t}(\Omega)$, we are interested in approximating $u \in H_{0}^{t}(\Omega)$ that solves

$$
a(u, v)=f(v) \quad\left(v \in H_{0}^{t}(\Omega)\right) .
$$

We set $\|\cdot\|:=a(\cdot, \cdot)^{\frac{1}{2}}$, being a norm on $H_{0}^{t}(\Omega)$ equivalent to $\|\cdot\|_{H^{t}(\Omega)}$. The corresponding operator norm on $\mathcal{L}\left(H_{0}^{t}(\Omega), H_{0}^{t}(\Omega)\right)$ will also be denoted as $\|\cdot\|$.

2.2. Multiplicative Schwarz method. Let $\Omega=\bigcup_{i=0}^{m-1} \Omega_{i}$ be some fixed decomposition of $\Omega$ into $m \in \mathbb{N}$ open sets, such that any $v \in H_{0}^{t}(\Omega)$ can be decomposed into $\sum_{i=0}^{m-1} v_{i}$ with $v_{i} \in H_{0}^{t}\left(\Omega_{i}\right)$ and, for some absolute constant $C_{0}$,

$$
\sum_{i=0}^{m-1}\left\|v_{i}\right\|_{H^{t}(\Omega)}^{2} \leq C_{0}\|v\|_{H^{t}(\Omega)}^{2} .
$$

With respect to this decomposition, the multiplicative Schwarz method for solving (2.1) reads as follows: With $u_{0}:=0$, for $k=1,2, \ldots$,

$$
u_{k}:=u_{k-1}+e_{k-1}
$$

where, with $i:=(k-1) \bmod m$,

$$
a\left(e_{k-1}, v\right)=f(v)-a\left(u_{k-1}, v\right) \quad\left(v \in H_{0}^{t}\left(\Omega_{i}\right)\right) .
$$

With $P_{i}: H_{0}^{t}(\Omega) \rightarrow H_{0}^{t}(\Omega)$ denoting the $a(\cdot, \cdot)$-orthogonal projector onto $H_{0}^{t}\left(\Omega_{i}\right)$, we have

It holds that

$$
u-u_{k}=\left(I-P_{i}\right)\left(u-u_{k-1}\right) .
$$

$$
\rho:=\left\|\left(I-P_{m-1}\right) \cdots\left(I-P_{0}\right)\right\|<1,
$$

only dependent on $C_{0}$ from (2.2) and the maximum number of subdomains that intersect with one subdomain (see Xu92; although the theory is formulated for finite dimensional Hilbert spaces, it extends directly to the infinite dimensional case).

We are going to study a version of this algorithm, in which the subdomain problems are solved only inexactly using adaptive wavelet methods. So we assume that for each $0 \leq i \leq m-1$, we have available a Riesz basis $\Psi^{(i)}=\left\{\psi_{\lambda}^{(i)}: \lambda \in \nabla^{(i)}\right\}$ for $H_{0}^{t}\left(\Omega_{i}\right)$ of wavelet type. Being a Riesz basis means that for any $v \in H_{0}^{t}\left(\Omega_{i}\right)$, the unique $\mathbf{v} \in \ell_{2}\left(\nabla^{(i)}\right)$ with $v=\mathbf{v}^{T} \Psi^{(i)}$ satisfies $\|v\|_{H^{t}\left(\Omega_{i}\right)} \approx\|\mathbf{v}\|_{\ell_{2}\left(\nabla^{(i)}\right)}$. 
From (2.2) one easily infers that $\Psi=\left\{\psi_{\lambda}: \lambda \in \nabla\right\}:=\bigcup_{i} \Psi^{(i)}$ is a frame for $H_{0}^{t}(\Omega)$, meaning that clos span $\Psi=H_{0}^{t}(\Omega)$ and, for some absolute constants $0<c_{1} \leq c_{2}$,

$$
c_{1}\|v\|_{H^{t}(\Omega)}^{2} \leq \inf _{\left\{\mathbf{v} \in \ell_{2}(\nabla): \mathbf{v}^{T} \Psi=v\right\}}\|\mathbf{v}\|_{\ell_{2}(\nabla)}^{2} \leq c_{2}\|v\|_{H^{t}(\Omega)}^{2} \quad\left(v \in H_{0}^{t}(\Omega)\right) .
$$

Due to the overlap between subdomains, note that generally the decomposition $v=\mathbf{v}^{T} \Psi$ is not unique.

The property of $\Psi$ being a frame with constants $c_{1}$ and $c_{2}$ is equivalent to

$$
c_{2}^{-1}\|g\|_{H^{-t}(\Omega)}^{2} \leq\left\|\left[g\left(\psi_{\lambda}\right)\right]_{\lambda \in \nabla}\right\|_{\ell_{2}(\nabla)}^{2} \leq c_{1}^{-1}\|g\|_{H^{-t}(\Omega)}^{2} \quad\left(g \in H^{-t}(\Omega)\right) .
$$

With $(A w)(v):=a(w, v), \mathbf{A}:=\left[a\left(\psi_{\mu}, \psi_{\lambda}\right)\right]_{\lambda, \mu \in \nabla}, \mathbf{f}:=\left[f\left(\psi_{\lambda}\right)\right]_{\lambda \in \nabla}$, we infer that

$$
\left\|u-\mathbf{v}^{T} \Psi\right\|_{H^{t}(\Omega)} \approx\left\|A\left(u-\mathbf{v}^{T} \Psi\right)\right\|_{H^{-t}(\Omega)} \approx\|\mathbf{f}-\mathbf{A v}\|_{\ell_{2}(\nabla)} \quad\left(\mathbf{v} \in \ell_{2}(\nabla)\right),
$$

which will be used in the numerical experiments to estimate errors.

Finally, in this section, we note that

$$
\|v\| \leq\|\mathbf{A}\|_{\ell_{2}(\nabla) \rightarrow \ell_{2}(\nabla)}^{\frac{1}{2}} \inf _{\left\{\mathbf{v} \in \ell_{2}(\nabla): \mathbf{v}^{T} \Psi=v\right\}}\|\mathbf{v}\|_{\ell_{2}(\nabla)} \quad\left(v \in H_{0}^{t}(\Omega)\right) .
$$

2.3. Wavelet assumptions. In the following (standard) wavelet assumptions are collected that will be used. We consider biorthogonal spline wavelets of order $d$ with $\frac{d-t}{n}>\frac{1}{2}$. The wavelets are assumed to be local in the sense that, with $|\lambda| \in \mathbb{N}_{0}$ denoting the level of the wavelet $\psi_{\lambda}^{(i)}$,

$$
\operatorname{diam}\left(\operatorname{supp} \psi_{\lambda}^{(i)}\right) \lesssim 2^{-|\lambda|}
$$

and

$$
\sup _{x \in \Omega_{i}, \ell \in \mathbb{N}_{0}} \#\left\{|\lambda|=\ell: B\left(x ; 2^{-\ell}\right) \cap \operatorname{supp} \psi_{\lambda}^{(i)} \neq \emptyset\right\}<\infty
$$

and that the dual wavelets $\left\{\tilde{\psi}_{\lambda}^{(i)}: \lambda \in \nabla^{(i)}\right\} \subset H^{-t}(\Omega)$, defined by $\tilde{\psi}_{\tilde{\lambda}}^{(i)}\left(\psi_{\lambda}^{(i)}\right)=$ $\delta_{\tilde{\lambda}, \lambda}$, are also local. Furthermore, it is assumed that the (primal) wavelets are piecewise smooth. That is, with $R$ being some absolute integer constant, we assume that $\operatorname{supp} \psi_{\lambda}^{(i)} \backslash \operatorname{sing} \operatorname{supp} \psi_{\lambda}^{(i)}$ is the disjoint union of domains $\Xi_{\lambda, 1}, \ldots, \Xi_{\lambda, R}$, with $\bigcup_{j=1}^{R} \overline{\Xi_{\lambda, j}}=\operatorname{supp} \psi_{\lambda}^{(i)}$, where $\left.\psi_{\lambda}^{(i)}\right|_{\Xi_{\lambda, j}}$ is smooth with, for any $\gamma \in \mathbb{N}_{0}^{n}$,

$$
\sup _{x \in \Xi_{\lambda, j}}\left|\partial^{\gamma} \psi_{\lambda}^{(i)}(x)\right| \lesssim 2^{|\lambda|\left(\frac{n}{2}+|\gamma|-t\right)}
$$

In addition, we require that there is a smooth, regular mapping $\kappa_{i}: \mathbb{R}^{n} \rightarrow \mathbb{R}^{n}$, such that $\kappa_{i}^{-1}\left(\Xi_{\lambda, j}\right)$ is an $n$-cube aligned with the Cartesian coordinates, and

$$
\left.\left(\psi_{\lambda}^{(i)} \circ \kappa_{i}\right)\right|_{\kappa_{i}^{-1}\left(\Xi_{\lambda, j}\right)} \in Q_{d-1},
$$

with $Q_{d-1}$ being the $n$-fold tensor product of the space of univariate polynomials of degree $d-1$.

Moreover, the wavelets will be chosen to have a maximal global smoothness, i.e.,

$$
\psi_{\lambda}^{(i)} \in C^{d-2}(\Omega)
$$

In particular, this condition means that on interior boundaries $\partial \Omega_{i} \cap \Omega$, homogeneous Dirichlet boundary conditions of the maximal order $d-2$ are incorporated in the wavelet construction. 
With the exception of wavelets on level 0 , we assume that all wavelets have $\mathbb{N}_{0} \ni \tilde{d}>d-2 t$ vanishing moments, meaning that, when $\tilde{d}>0$,

$$
\psi_{\lambda}^{(i)} \circ \kappa_{i} \perp P_{\tilde{d}-1} .
$$

Finally, we will assume that, in relation to the size of the minimal overlap between subdomains, the diameters of the supports of all primal and dual wavelets are sufficiently small, i.e., that the "initial meshes" are sufficiently fine. A precise formulation of this assumption will be given in Theorem 3.4.

Starting from biorthogonal spline multiresolution analyses on the line (CDF92]), using tensor products, wavelets satisfying above assumptions can be constructed on $(0,1)^{n}$ ([DS98] $)$, after which they can be lifted to $\Omega_{i}:=\kappa_{i}\left((0,1)^{n}\right)$.

2.4. Approximation classes. For $s>0$, let the approximation class $\mathcal{A}_{\infty}^{s}\left(\nabla^{(i)}\right):=$ $\left\{\mathbf{v} \in \ell_{2}\left(\nabla^{(i)}\right):\|\mathbf{v}\|_{\mathcal{A}_{\infty}^{s}\left(\nabla^{(i)}\right)}<\infty\right\}$, where

$$
\|\mathbf{v}\|_{\mathcal{A}_{\infty}^{s}\left(\nabla^{(i)}\right)}:=\sup _{\varepsilon>0} \varepsilon \times\left[\min \left\{N \in \mathbb{N}_{0}:\left\|\mathbf{v}-\mathbf{v}_{N}\right\|_{\ell_{2}\left(\nabla^{(i)}\right)} \leq \varepsilon\right\}\right]^{s},
$$

and $\mathbf{v}_{N}$ denotes a best $N$-term approximation for $\mathbf{v}$, i.e., a vector in $\ell_{2}\left(\nabla^{(i)}\right)$ that agrees with $\mathbf{v}$ on the locations where the latter has its $N$ largest values in modulus, and which is zero elsewhere. For $\tau \in(0,2)$, the space $\ell_{\tau, \infty}\left(\nabla^{(i)}\right)$ (also known as weak $\left.\ell_{\tau}\left(\nabla^{(i)}\right)\right)$ is defined by

$$
\ell_{\tau, \infty}\left(\nabla^{(i)}\right):=\left\{\mathbf{v} \in \ell_{2}\left(\nabla^{(i)}\right):\|\mathbf{v}\|_{\ell_{\tau, \infty}\left(\nabla^{(i)}\right)}:=\sup _{k \in \mathbb{N}} k^{1 / \tau}\left|\gamma_{k}(\mathbf{v})\right|<\infty\right\}
$$

where $\left|\gamma_{k}(\mathbf{v})\right|$ denotes the $k$ th largest coefficient in modulus of $\mathbf{v}$. It is known that for each $s>0$,

$$
\|\mathbf{v}\|_{\mathcal{A}_{\infty}^{s}\left(\nabla^{(i)}\right)} \approx\|\mathbf{v}\|_{\ell_{\tau, \infty}\left(\nabla^{(i)}\right)}
$$

where, here and in the remainder of this paper, $s$ and $\tau$ are always related according to

$$
\tau=\left(\frac{1}{2}+s\right)^{-1} .
$$

We define $\mathcal{A}_{\infty}^{s}(\nabla)$ and $\ell_{\tau, \infty}(\nabla)$ similarly as for the index sets $\nabla^{(i)}$.

\section{The Adaptive SchWarz Method And its optimality}

3.1. Schwarz method with inexact subdomain solves. Our aim is to construct a numerical method that has the following property:

If for some $s>0$, the solution $u$ of (2.1) has some representation $u=\tilde{\mathbf{u}}^{T} \Psi$ with $\tilde{\mathbf{u}} \in \ell_{\tau, \infty}(\nabla)$, then for any $\varepsilon>0$ the method produces a $\mathbf{u}_{\varepsilon} \in \ell_{2}(\nabla)$ with $\left\|u-\mathbf{u}_{\varepsilon}^{T} \Psi\right\|_{H^{t}(\Omega)} \lesssim \varepsilon$ and \# $\operatorname{supp} \mathbf{u}_{\varepsilon} \lesssim \varepsilon^{-1 / s}\|\tilde{\mathbf{u}}\|_{\ell_{\tau, \infty}(\nabla)}^{1 / s}$, taking a number of operations that can be bounded by the same expression.

Note that if we would have best $N$-term approximations available, then in order to guarantee $\left\|\tilde{\mathbf{u}}-\tilde{\mathbf{u}}_{N}\right\| \leq \varepsilon$, and with that $\left\|u-\tilde{\mathbf{u}}_{N}^{T} \Psi\right\|_{H^{t}(\Omega)} \lesssim \varepsilon$, generally $N=$ $\varepsilon^{-1 / s}\|\tilde{\mathbf{u}}\|_{\mathcal{A}_{\infty}^{s}(\nabla)}^{1 / s} \approx \varepsilon^{-1 / s}\|\tilde{\mathbf{u}}\|_{\ell_{\tau, \infty}(\nabla)}^{1 / s}$ is required. In this sense, such a method can be called (quasi-) optimal.

Remark 3.1. As we will see in Section 5. assuming a strictly positive overlap between subdomains (cf. (3.4) ), a representation $\tilde{\mathbf{u}} \in \ell_{\tau, \infty}(\nabla)$ exists if and only if $u$ is contained in a certain Banach space $\mathcal{B}^{s}(\Omega)$, with $B_{\tau, \tau}^{s n+t}(\Omega) \cap H_{0}^{t}(\Omega) \hookrightarrow$ $\mathcal{B}^{s}(\Omega) \hookrightarrow H_{0}^{t}(\Omega)$. The space $B_{\tau, \tau}^{s n+t}(\Omega) \cap H_{0}^{t}(\Omega)$, and so $\mathcal{B}^{s}(\Omega)$ is larger than the space $H^{s n+t}(\Omega) \cap H_{0}^{t}(\Omega)$, with an increasing difference with growing $s$. Recall that 
$u$ has to be in $H^{s n+t}(\Omega) \cap H_{0}^{t}(\Omega)$ in order to ensure the same rate of approximation with a standard linear approximation scheme of order $d$. The statements in this remark are equally valid when $m=1$, in which case $\Psi$ is a wavelet basis for $H_{0}^{t}(\Omega)$.

In view of the aforementioned aim, considering the multiplicative Schwarz method, it is clear that the auxiliary problems (2.3) on the subdomains should be solved only inexactly. In any case if we apply linearly decreasing tolerances as the iteration proceeds, using (2.4) it is easily seen that then we still have majorized linear convergence of the iterands towards $u$.

Let us write the $k$ th iterand $u_{k}=\sum_{i=0}^{m-1} u_{k}^{(i)}$ with $u_{k}^{(i)}=\left(\mathbf{u}_{k}^{(i)}\right)^{T} \Psi^{(i)}$. Then, with the canonical approach that an approximate solve on subdomain $\Omega_{i}$ amounts to an update of the expansion coefficients with respect to $\Psi^{(i)}$ only, for $k \in i+1+m \mathbb{N}_{0}$ we have

$$
u_{k}^{(i)}-u_{k-m}^{(i)}=u_{k}-u_{k-1} .
$$

From the majorized linear convergence of $\left(u_{k}\right)_{k}$, we infer that $\left(u_{k}^{(i)}\right)_{k \in i+1+m \mathbb{N}_{0}}$ is a Cauchy sequence in $H_{0}^{t}(\Omega)$, and thus convergent to some $u^{(i)} \in H_{0}^{t}(\Omega)$ with representation $\left(\mathbf{u}^{(i)}\right)^{T} \Psi^{(i)}$. With $\varepsilon_{k}$ denoting the linearly decreasing upper bound for $\left\|u-u_{k}\right\|_{H^{t}(\Omega)}$, we have $\left\|\mathbf{u}^{(i)}-\mathbf{u}_{k}^{(i)}\right\|_{\ell_{2}\left(\nabla^{(i)}\right)} \approx\left\|u^{(i)}-u_{k}^{(i)}\right\|_{H^{t}\left(\Omega_{i}\right)} \lesssim \varepsilon_{k}$. So the requirement that for any $\varepsilon>0$, \# $\operatorname{supp} \mathbf{u}_{k} \lesssim \varepsilon^{-1 / s}\|\tilde{\mathbf{u}}\|_{\ell_{\tau, \infty}(\nabla)}^{1 / s}$ whenever the algorithm is stopped as soon as $\varepsilon_{k} \leq \varepsilon$ can only be fulfilled when each of the $\mathbf{u}^{(i)} \in \ell_{\tau, \infty}\left(\nabla^{(i)}\right)$ with $\left\|\mathbf{u}^{(i)}\right\|_{\ell_{\tau, \infty}\left(\nabla^{(i)}\right)} \lesssim\|\tilde{\mathbf{u}}\|_{\ell_{\tau, \infty}(\nabla)}$.

With the above "canonical" approach we do not know why this necessary (and, as we will see, sufficient) condition for optimality would be satisfied. Therefore, we consider a modified approach, where in each iteration not only the expansion coefficients corresponding to the current subdomain are updated, but also those corresponding to neighboring subdomains. The fact that such a modification is allowed is based on the observation that if before solving on subdomain $\Omega_{i}$, some function from $H_{0}^{t}\left(\Omega_{i}\right)$ is subtracted from the current iterand, then this has no influence on the iterand after the (exact) solving on $\Omega_{i}$. This can be easily deduced from (2.3). We will show that with a suitable modified approach, the $\mathbf{u}_{k}^{(i)}$ converge to some $\mathbf{u}^{(i)}$ with $\left\|\mathbf{u}^{(i)}\right\|_{\ell_{\tau, \infty}\left(\nabla^{(i)}\right)} \lesssim\|\tilde{\mathbf{u}}\|_{\ell_{\tau, \infty}(\nabla)}$. By adding a so-called coarsening routine to our algorithm, we show that this property guarantees optimality.

We will make use of a routine $\mathbf{C O A R S E}[\mathbf{v}, \varepsilon] \rightarrow \mathbf{w}_{\varepsilon}$ that for $\varepsilon>0$ and $\mathbf{w} \in$ $\ell_{0}(\nabla)$, being the space of sequences with finite support, determines a $\mathbf{w}_{\varepsilon} \in \ell_{0}(\nabla)$, such that

$$
\left\|\mathbf{w}-\mathbf{w}_{\varepsilon}\right\|_{\ell_{2}(\nabla)} \leq \varepsilon
$$

and

$$
\# \operatorname{supp} \mathbf{w}_{\varepsilon} \lesssim \min \left\{N:\left\|\mathbf{w}-\mathbf{w}_{N}\right\|_{\ell_{2}(\nabla)} \leq \varepsilon\right\} .
$$

The recurrent application of coarsening inside adaptive iterative solvers was first proposed in CDD01. It removes small coefficients from an approximation vector, with which an optimal balance is retained between support length and accuracy, as is demonstrated in the forthcoming Proposition 3.2 .

Since $\mathbf{w}$ is finitely supported, we could select $\mathbf{w}_{\varepsilon}$ to be $\mathbf{w}_{N}$ for the smallest $N$ with $\left\|\mathbf{w}-\mathbf{w}_{N}\right\|_{\ell_{2}(\nabla)} \leq \varepsilon$. This, however, would require a sorting of the coefficients of $\mathbf{w}$ by their modulus taking $\mathcal{O}(\# \operatorname{suppw} \log (\#$ suppw $))$ operations. In order to avoid the log factor, we apply an approximate bucket sorting (see Bar05, Ste03. 
for details) at the expense of losing in (3.1) some factor larger than 1 that one can choose. Now the number of operations is bounded by an absolute multiple of

$$
\# \operatorname{supp} \mathbf{w}+\max \left(\log \left(\varepsilon^{-1}\|\mathbf{w}\|_{\ell_{2}(\nabla)}\right), 1\right) \text {. }
$$

In view of the cost of our approximate multiplicative Schwarz routine, we note that the above $\log$ term turns out to be harmless, because for any $s>0, \log \left(\varepsilon^{-1}\|\mathbf{w}\|_{\ell_{2}(\nabla)}\right)$ $\lesssim \varepsilon^{-1 / s}\|\mathbf{w}\|_{\ell_{2}(\nabla)}^{1 / s}$.

The following proposition shows the benefit of the application of coarsening. A proof can be found in e.g. Coh03, Theorem 4.9.1], [DFR ${ }^{+}$07, Proposition 3.4].

Proposition 3.2. Let $\zeta>1$ and $s>0$. Then for any $\varepsilon>0, \mathbf{v} \in \ell_{\tau, \infty}(\nabla)$ and $\mathbf{w} \in \ell_{0}(\nabla)$ with

$$
\|\mathbf{v}-\mathbf{w}\|_{\ell_{2}(\nabla)} \leq \varepsilon
$$

for $\mathbf{w}_{\zeta \varepsilon}:=\operatorname{COARSE}[\zeta \varepsilon, \mathbf{w}]$ we have that

$$
\# \operatorname{supp} \mathbf{w}_{\zeta \varepsilon} \lesssim \varepsilon^{-1 / s}\|\mathbf{v}\|_{\ell_{\tau, \infty}(\nabla)}^{1 / s}, \quad\left\|\mathbf{w}_{\zeta \varepsilon}\right\|_{\ell_{\tau, \infty}(\nabla)} \lesssim\|\mathbf{v}\|_{\ell_{\tau, \infty}(\nabla)},
$$

and obviously, $\left\|\mathbf{v}-\mathbf{w}_{\zeta \varepsilon}\right\|_{\ell_{2}(\nabla)} \leq(1+\zeta)\|\mathbf{v}-\mathbf{w}\|_{\ell_{2}(\nabla)}$.

We are ready to formulate our multiplicative Schwarz method with inexact subdomain solves. It consists of three nested loops, where in the innermost loop a complete multiplicative Schwarz iteration is performed, i.e., the subsequent (inexact) solving of the local problems on all $m$ subdomains. After $K$ multiplicative Schwarz iterations, coarsening is applied which happens in the middle loop. In the outermost loop, the whole process is repeated $L$ times to guarantee a desired reduction of the initial error.

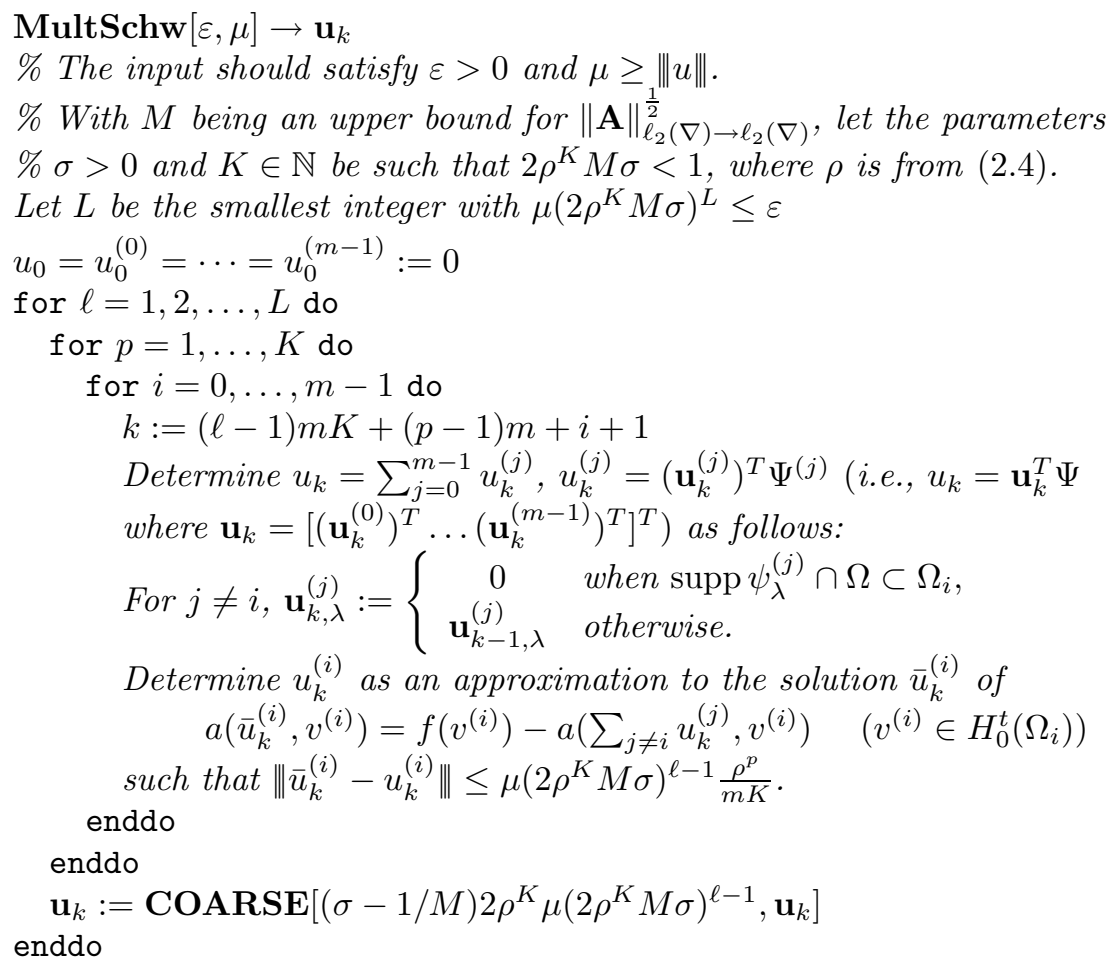


Note that for $k \in m K \mathbb{N}, \mathbf{u}_{k}$ (and thus also $u_{k}$ ) got redefined by the application of COARSE, i.e., for those $k, u_{k}$ or $\mathbf{u}_{k}$ has two meanings that will be distinguished carefully.

Referring to the preceding discussion, note that before solving on subdomain $\Omega_{i}$, the current iterand $u_{k-1}$ is modified by subtracting functions from $H_{0}^{t}\left(\Omega_{i}\right)$. So apart from the fact that the problems on the subdomains are solved inexactly, and that a coarsening routine is added, the algorithm is an implementation of the multiplicative Schwarz method.

\subsection{Convergence.}

Proposition 3.3. Let $\mu \geq\|u\|$. The iterand $u_{\ell m K}$ inside $\mathbf{u}_{\varepsilon}:=\operatorname{MultSchw}[\varepsilon, \mu]$, for $\ell>0$ after the application of COARSE, satisfies

$$
\left\|u-u_{\ell m K}\right\| \leq \mu\left(2 \rho^{K} M \sigma\right)^{\ell},
$$

and so $\left\|u-\mathbf{u}_{\varepsilon}^{T} \Psi\right\| \leq \varepsilon$. Furthermore, for $1 \leq q \leq m K$,

$$
\left\|u-u_{(\ell-1) m K+q}\right\| \leq 2 \mu\left(2 \rho^{K} M \sigma\right)^{\ell-1} \rho^{\frac{q}{m}-1},
$$

where for $q=m K, u_{\ell m K}$ should read here as the iterand before the application of COARSE.

Proof. The first statement is valid for $\ell=0$. Suppose it is valid for some $\ell-1 \geq 0$. Then for $p=0, \ldots, K$,

$$
\left\|u-u_{(\ell-1) m K+p m}\right\| \leq \mu\left(1+\frac{p}{K}\right) \rho^{p}\left(2 \rho^{K} M \sigma\right)^{\ell-1},
$$

where, when $p=K, u_{\ell m K}$ should read here as the iterand before the application of COARSE. Indeed, this estimate is valid for $p=0$. Suppose it is valid for some $p-1 \geq 0$. Then (2.4) and $\left\|I-P_{i}\right\| \leq 1$ show that

$$
\begin{aligned}
\left\|u-u_{(\ell-1) m K+p m}\right\| \leq & \rho\left[\mu\left(1+\frac{p-1}{K}\right) \rho^{p-1}\left(2 \rho^{K} M \sigma\right)^{\ell-1}\right] \\
& +m \mu\left(2 \rho^{K} M \sigma\right)^{\ell-1} \frac{\rho^{p}}{m K}
\end{aligned}
$$

which is 3.3 .

By (3.3) for $p=K$, and the fact that $\left\|\mathbf{v}^{T} \Psi\right\| \leq M\|\mathbf{v}\|_{\ell_{2}(\nabla)}\left(\mathbf{v} \in \ell_{2}(\nabla)\right)$ by definition of $M$ (cf. (2.5) ), the subsequent application of COARSE introduces an additional error that in $\|\cdot\|$ is not larger than $M(\sigma-1 / M) 2 \rho^{K} \mu\left(2 \rho^{K} M \sigma\right)^{\ell-1}$, the proof of the first statement is completed.

From (3.3), with $p$ reading as $p-1$, and $\left\|I-P_{i}\right\| \leq 1$, we find that

$$
\left\|u-u_{(\ell-1) m K+(p-1) m+(i+1)}\right\| \leq\left[1+\frac{p-1}{K}+\frac{(i+1) \rho}{m K}\right] \rho^{p-1} \mu\left(2 \rho^{K} M \sigma\right)^{\ell-1},
$$

where here when $p=K$ and $i=m-1, u_{\ell m K}$ should read as the iterand before the application of COARSE. From this estimate and $1+\frac{p-1}{K}+\frac{(i+1) \rho}{m K} \leq 2$, the second statement of the theorem follows easily.

3.3. Optimality. Having established convergence of MultSchw, below we investigate its optimality. For $A \subset \mathbb{R}^{n}$ and $\delta \geq 0$, let $B(A ; \delta):=\left\{x \in \mathbb{R}^{n}: \operatorname{dist}(x, A) \leq \delta\right\}$, and

$$
\Omega_{i}(-\delta):=\left\{x \in \Omega_{i}: B(x ; \delta) \cap \Omega \subset \Omega_{i}\right\} .
$$

In Sections 4 and 5 , we will prove the following result. 
Theorem 3.4. Let the decomposition $\Omega=\bigcup_{i=0}^{m-1} \Omega_{i}$ be such that

$$
\Omega \subset \bigcup_{i=0}^{m-1} \Omega_{i}(-\omega) \text { for some } \omega>0
$$

and let

$$
\eta:=\max _{0 \leq i \leq m-1, \lambda \in \nabla^{(i)}}\left(\operatorname{diam}\left(\operatorname{supp} \psi_{\lambda}^{(i)}\right), \operatorname{diam}\left(\operatorname{supp} \tilde{\psi}_{\lambda}^{(i)}\right)\right) \leq \frac{\omega}{3 m-1} .
$$

(a) Then, for any $0 \leq i \leq m-1$, there exists a $u^{(i)}=\left(\mathbf{u}^{(i)}\right)^{T} \Psi^{(i)} \in H_{0}^{t}\left(\Omega_{i}\right)$ such that for $\ell \in \mathbb{N}, 1 \leq q \leq m K$, with $(q-1) \bmod m=i$,

$$
\left\|u^{(i)}-u_{(\ell-1) m K+q}^{(i)}\right\|_{H^{t}\left(\Omega_{i}\right)} \lesssim \mu\left(2 \rho^{K} M \sigma\right)^{\ell-1} \rho^{\frac{q}{m}-1},
$$

only dependent on $m, \omega$ and the Riesz constants of all $\Psi^{(i)}$, and where for $q=m K$, $u_{\ell m K}$ should read as the iterand before the application of COARSE.

(b) If, for some $s<\frac{d-t}{n}$, u has some representation $\tilde{\mathbf{u}}^{T} \Psi$ with $\tilde{\mathbf{u}} \in \ell_{\tau, \infty}(\nabla)$, then $\left\|\mathbf{u}^{(i)}\right\|_{\ell_{\tau, \infty}\left(\nabla^{(i)}\right)} \lesssim\|\tilde{\mathbf{u}}\|_{\ell_{\tau, \infty}(\nabla)}$.

Remark 3.5. Concerning the restriction $s<\frac{d-t}{n}$, note that in view of the polynomial order of approximation being applied, even a $C^{\infty}$ solution $u$ cannot be expected to have a representation $\tilde{\mathbf{u}}^{T} \Psi$ with $\tilde{\mathbf{u}} \in \ell_{\tau, \infty}(\nabla)$ for $s>\frac{d-t}{n}$. Unfortunately, interpolation arguments that are going to be applied in Section 5 do not allow us to cover the case $s=\frac{d-t}{n}$.

Remark 3.6. The condition (3.4) implies (2.2), but the converse is not true as is demonstrated by an example from $\left[\mathrm{DFR}^{+} 07, \S 4.2\right]$. The condition (3.5) means that the minimal overlap between subdomains is sufficiently large compared to the largest diameter of the support of any primal or dual wavelet.

We will apply Theorem 3.4 in two ways. First and most importantly, for some $s<\frac{d-t}{n}$, let $u$ have some representation $\tilde{\mathbf{u}}^{T} \Psi$ with $\tilde{\mathbf{u}} \in \ell_{\tau, \infty}(\nabla)$, and let $\mathbf{u} \in \ell_{2}(\nabla)$ be defined by

$$
\mathbf{u}_{\lambda}=\left\{\begin{array}{cl}
\mathbf{u}_{\lambda}^{(i)} & \text { when } \lambda \in \nabla^{(i)} \text { and } \operatorname{supp} \psi_{\lambda}^{(i)} \cap \Omega \not \subset \Omega_{j} \text { for some } j>i, \\
0 & \text { otherwise. }
\end{array}\right.
$$

From Theorem 3.4(b), we infer that

$$
\|\mathbf{u}\|_{\ell_{\tau, \infty}(\nabla)} \lesssim\|\tilde{\mathbf{u}}\|_{\ell_{\tau, \infty}(\nabla)}
$$

Because $u_{\ell m K}$ before COARSE is given by

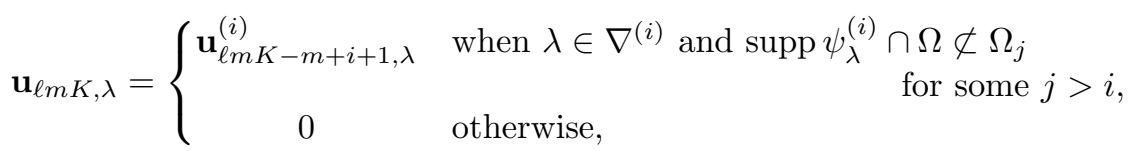

by summing the bound (3.6) over $m K-m+1 \leq q \leq m K$, and using that the $\Psi^{(i)}$ are Riesz bases, we find that

$$
\left\|\mathbf{u}-\mathbf{u}_{\ell m K}\right\|_{\ell_{2}(\nabla)} \leq \mu C\left(2 \rho^{K} M \sigma\right)^{\ell-1} \rho^{K-2},
$$

for some constant $C>0$ only dependent on $m, \omega$ and the Riesz constants of all $\Psi^{(i)}$. Note that in particular this result, in combination with Proposition 3.3, shows that $u=\mathbf{u}^{T} \Psi$. 
In view of Proposition 3.2 we conclude that if $\sigma$ was chosen to be sufficiently large in the sense that

$$
(\sigma-1 / M) 2>C \rho^{-2}
$$

(and $K$ correspondingly such that $2 \rho^{K} M \sigma<1$ ), then after evaluation of $\mathbf{u}_{\ell m K}:=$ COARSE $\left[(\sigma-1 / M) 2 \rho^{K} \mu\left(2 \rho^{K} M \sigma\right)^{\ell-1}, \mathbf{u}_{\ell m K}\right]$ inside the routine MultSchw, we have

$$
\# \operatorname{supp} \mathbf{u}_{\ell m K} \lesssim\left[\mu\left(2 \rho^{K} M \sigma\right)^{\ell}\right]^{-1 / s}\|\tilde{\mathbf{u}}\|_{\ell_{\tau, \infty}(\nabla)}^{1 / s}
$$

and

$$
\left\|\mathbf{u}_{\ell m K}\right\|_{\ell_{\tau, \infty}(\nabla)} \lesssim\|\tilde{\mathbf{u}}\|_{\ell_{\tau, \infty}(\nabla)}
$$

uniformly in $\ell$. In the remainder we will assume that such a choice of $\sigma$ and $K$ has been made.

With a suitable implementation of the inexact solves on the subdomains, the estimates (3.8) and (3.9) are the key to a proof of optimality of MultSchw. Since the arguments follow by now standard lines for adaptive wavelet methods (cf. CDD01, CDD02, Ste03, SW08), we only sketch the main steps:

A second application of Theorem 3.4 (part (a)) shows that if for approximating the solution $\bar{u}_{k}^{(i)}$ on subdomain $\Omega_{i}$, we use the previous iterand $u_{k-m}^{(i)}$ as the starting value of an iterative solver, then we have to reduce its error by a constant factor only.

For bounding the cost of doing so we have to make some assumptions on the cost of approximating the infinite "load vector" f and that of the approximate application of the bi-infinite "stiffness matrix" $\mathbf{A}$.

For some $s^{*} \geq \frac{d-t}{n}$, we assume that $\mathbf{A}$ is $s^{*}$-admissible, meaning that there exists an approximate matrix vector routine $\mathbf{A P P L Y}[\mathbf{w}, \varepsilon] \rightarrow \mathbf{z}_{\varepsilon}$, taking a $\mathbf{w} \in \ell_{0}(\nabla)$, and producing $\mathbf{z}_{\varepsilon} \in \ell_{0}(\nabla)$, that has the following properties (cf. CDD02, Ste03]):

(1) $\left\|\mathbf{A} \mathbf{w}-\mathbf{z}_{\varepsilon}\right\|_{\ell_{2}(\nabla)} \leq \varepsilon$.

(2) For any $s<s^{*}, \# \operatorname{supp} \mathbf{z}_{\varepsilon} \lesssim \varepsilon^{-1 / s}\|\mathbf{w}\|_{\ell_{\tau, \infty}(\nabla)}^{1 / s}$ and the number of operations required by the call is bounded by some absolute multiple of $1+\#$ supp w + $\varepsilon^{-1 / s}\|\mathbf{w}\|_{\ell_{\tau, \infty}(\nabla)}^{1 / s}$.

As consequences, for $s<s^{*}$ it holds that

$$
\begin{aligned}
& \left\|\mathbf{z}_{\varepsilon}\right\|_{\ell_{\tau, \infty}(\nabla)} \lesssim\|\mathbf{w}\|_{\ell_{\tau, \infty}(\nabla)} \quad\left(\mathbf{w} \in \ell_{0}(\nabla), \varepsilon>0\right) \text { and } \\
& \mathbf{A}: \ell_{\tau, \infty}(\nabla) \rightarrow \ell_{\tau, \infty}(\nabla) \quad \text { is bounded. }
\end{aligned}
$$

Concerning $\mathbf{f}$, we assume that we have a routine $\mathbf{R H S}[\varepsilon] \rightarrow \mathbf{f}_{\varepsilon}$ available, that is quasi-optimal in the following sense:

(3) $\left\|\mathbf{f}-\mathbf{f}_{\varepsilon}\right\|_{\ell_{2}(\nabla)} \leq \varepsilon$.

(4) \# $\operatorname{supp} \mathbf{f}_{\varepsilon} \lesssim \min \left\{N:\left\|\mathbf{f}-\mathbf{f}_{N}\right\|_{\ell_{2}(\nabla)} \leq \varepsilon\right\}$, and the cost of producing $\mathbf{f}_{\varepsilon}$ can be bounded on an absolute multiple of its support length +1 . 
As consequences, if, for some $s \in\left(0, s^{*}\right), u$ has some representation $\tilde{\mathbf{u}}^{T} \Psi$ with $\tilde{\mathbf{u}} \in \ell_{\tau, \infty}(\nabla)$, then from (3.11), (4) and (3) it follows that

$$
\# \operatorname{supp} \mathbf{f}_{\varepsilon} \lesssim \varepsilon^{-1 / s}\|\tilde{\mathbf{u}}\|_{\ell_{\tau, \infty}(\nabla)}^{1 / s}
$$

the number of operations required by the computation of $\mathbf{f}_{\varepsilon}$

is bounded by some absolute multiple of $1+\varepsilon^{-1 / s}\|\tilde{\mathbf{u}}\|_{\ell_{\tau, \infty}(\nabla)}^{1 / s}$,

$$
\left\|\mathbf{f}_{\varepsilon}\right\|_{\ell_{\tau, \infty}(\nabla)} \lesssim\|\tilde{\mathbf{u}}\|_{\ell_{\tau, \infty}(\nabla)} \quad(\varepsilon>0) .
$$

With $\mathbf{A}^{(i, j)}:=\left[a\left(\psi_{\mu}^{(j)}, \psi_{\lambda}^{(i)}\right)\right]_{\lambda \in \nabla^{(i)}, \mu \in \nabla^{(j)}}$, the matrix $\mathbf{A}$ has a natural partition as $\left[\mathbf{A}^{(i, j)}\right]_{0 \leq i, j \leq m-1}$. For $k:=(\ell-1) m K+(p-1) m+i+1$, the determination of $u_{k}^{(i)}$ inside MultSchw amounts to approximating the solution $\overline{\mathbf{u}}_{k}^{(i)}$ of

$$
\mathbf{A}^{(i, i)} \overline{\mathbf{u}}_{k}^{(i)}=\left.\mathbf{f}\right|_{\nabla^{(i)}}-\sum_{j \neq i} \mathbf{A}^{(i, j)} \mathbf{u}_{k}^{(j)}
$$

within tolerance $\delta:=\mu\left(2 \rho^{K} M \sigma\right)^{\ell-1} \frac{\rho^{p}}{m K}$ in $\|\cdot\|:=\left\langle\mathbf{A}^{(i, i)},, \cdot\right\rangle_{\ell_{2}\left(\nabla^{(i)}\right)}^{\frac{1}{2}} \bar{\sim}\|\cdot\|_{\ell_{2}\left(\nabla^{(i)}\right)}$. This can be realized by first approximating both parts of the right-hand side of this equation within tolerance $\left\|\left(\mathbf{A}^{(i, i)}\right)^{-1}\right\|_{\ell_{2}\left(\nabla^{(i)}\right) \rightarrow \ell_{2}\left(\nabla^{(i)}\right)}^{-\frac{1}{2}} \delta / 4$ in $\|\cdot\|_{\ell_{2}\left(\nabla^{(i)}\right)}$. Then we have $\left\|\overline{\mathbf{u}}_{k}^{(i)}-\left(\mathbf{A}^{(i, i)}\right)^{-1} \mathbf{g}\right\| \leq \delta / 2$ where $\mathbf{g}$ denotes the resulting approximation of the right-hand side. Since $\mathbf{A}^{(i, i)}$ is symmetric and positive definite, there exists a damping parameter $\alpha$ such that the correspondingly damped Richardson iteration for approximating $\left(\mathbf{A}^{(i, i)}\right)^{-1} \mathbf{g}$ converges in $\|\cdot\|$ with a rate $\xi<1$. As we have seen, from the previous solve on subdomain $\Omega_{i}$, we have available a vector $\mathbf{u}_{k-m}^{(i)}$ with $\left\|\overline{\mathbf{u}}_{k}^{(i)}-\mathbf{u}_{k-m}^{(i)}\right\| \leq D \delta$ for some constant $D>0$, and thus $\left\|\left(\mathbf{A}^{(i, i)}\right)^{-1} \mathbf{g}-\mathbf{u}_{k-m}^{(i)}\right\| \leq$ $\left(D+\frac{1}{2}\right) \delta$. Let $P$ be the smallest integer with $2 \xi^{P}\left(D+\frac{1}{2}\right) \leq \frac{1}{2}$. Then if we apply $P$ steps of damped Richardson iteration, where in the $q$ th iteration the application of $\mathbf{A}^{(i, i)}$ onto the current iterand is approximated within tolerance $\left(\xi^{q}\left(D+\frac{1}{2}\right) \delta\right) / P$ in $\|\cdot\|$, we end up with an approximation $\mathbf{u}_{k}^{(i)}$ to $\left(\mathbf{A}^{(i, i)}\right)^{-1} \mathbf{g}$ within tolerance $\frac{1}{2} \delta$ in $\|\cdot\|$, so that $\left\|\overline{\mathbf{u}}_{k}^{(i)}-\mathbf{u}_{k}^{(i)}\right\| \leq \delta$ as required.

Since the described intermediate steps to get from $\mathbf{u}_{(\ell-1) m K}$ to $\mathbf{u}_{\ell m K}$ involve a fixed number of approximate applications of the $\mathbf{A}^{(i, j)}$ by calls of APPLY, and a fixed number of approximations of the $\left.\mathbf{f}\right|_{\nabla^{(i)}}$ by calls of RHS, from (3.9), (3.10) and (3.14), we infer that $\left\|\mathbf{u}_{k}\right\|_{\ell_{\tau, \infty}(\nabla)} \lesssim\|\tilde{\mathbf{u}}\|_{\ell_{\tau, \infty}(\nabla)}$ uniformly in $k$. Taking into account the majorized linearly decreasing tolerances with which these calls are made, from (3.8), (2) and (3.12) we infer that

$$
\# \operatorname{supp} \mathbf{u}_{k} \lesssim\left[\mu\left(2 \rho^{K} M \sigma\right)^{k / m K}\right]^{-1 / s}\|\tilde{\mathbf{u}}\|_{\ell_{\tau, \infty}(\nabla)}^{1 / s},
$$

uniformly in $k$. Furthermore, if $\mu \lesssim\|u\|$, then (2), (3.13) and the discussion about the cost of a call of COARSE preceding Proposition 3.2. show that the number of operations required for the computation of $\mathbf{u}_{k}$ can be bounded by some absolute multiple of $\left[\mu\left(2 \rho^{K} M \sigma\right)^{k / m K}\right]^{-1 / s}\|\tilde{\mathbf{u}}\|_{\ell_{\tau, \infty}(\nabla)}^{1 / s}$. Here the assumption $\mu \lesssim\|u\|$, that implies $1 \lesssim \mu^{-1 / s}\|\tilde{\mathbf{u}}\|_{\ell_{\tau, \infty}(\nabla)}^{1 / s}$, is made to ensure that the constant term in the bounds for the cost of calls of APPLY and RHS never dominates the other terms. Since on the other hand $\left\|u-u_{k}\right\| \lesssim \mu\left(2 \rho^{K} M \sigma\right)^{k / m K}\left(k \in \mathbb{N}_{0}\right)$, as was shown in (3.2), we end up with the following optimality result. 
Theorem 3.7. Assume (3.4) and (3.5). Let $\sigma$ be sufficiently large such that (3.7) is valid, and let $K \in \mathbb{N}$ be such that $2 \rho^{K} M \sigma<1$. For some $s^{*} \geq \frac{d-t}{n}$, let $\mathbf{A}$ be $s^{*}$ admissible and RHS be quasi-optimal. Let u have some representation $\tilde{\mathbf{u}}^{T} \Psi$ with $\tilde{\mathbf{u}} \in \ell_{\tau, \infty}(\nabla)$ for some $s \in\left(0, \frac{d-t}{n}\right)$. Then for $\varepsilon \in(0, \mu), \mathbf{u}_{\varepsilon}:=\operatorname{MultSchw}[\varepsilon, \mu]$ satisfies $\left\|u-\mathbf{u}_{\varepsilon}^{T} \Psi\right\| \leq \varepsilon$ and \# $\operatorname{supp} \mathbf{u}_{\varepsilon} \lesssim \varepsilon^{-1 / s}\|\tilde{\mathbf{u}}\|_{\ell_{\tau, \infty}(\nabla)}^{1 / s}$, where the number of operations required by the call can be bounded on some absolute multiple of $\varepsilon^{-1 / s}\|\tilde{\mathbf{u}}\|_{\ell_{\tau, \infty}(\nabla)}^{1 / s}$.

A sufficient condition for $\mathbf{A}$ being $s^{*}$-admissible is that it can be sufficiently well approximated by computable sparse matrices quantified by the concept of $s^{*}$ computability (cf. CDD02, Ste03]). A matrix A is called to be $s^{*}$-computable when for all $N \in \mathbb{N}$, there is an infinite matrix $\mathbf{A}_{N}$, having in each column $\mathcal{O}(N)$ non-zero entries whose computations require $\mathcal{O}(N)$ operations, such that the error $\left\|\mathbf{A}-\mathbf{A}_{N}\right\|_{\ell_{2}(\nabla) \rightarrow \ell_{2}(\nabla)} \lesssim N^{-s}$ for any $s<s^{*}$.

For $a(\cdot, \cdot)$ resulting from a variational formulation of a boundary value problem with a partial differential operator of order $2 t$ having smooth coefficients, and homogeneous Dirichlet boundary conditions of order $t-1$, and for wavelets on the subdomains that satisfy the assumptions collected in Section 2.3, in [SW08, it was shown that indeed $\mathbf{A}$ is $s^{*}$-computable for some $s^{*} \geq \frac{d-t}{n}$.

Generally, the existence of a quasi-optimal RHS routine depends on the righthand side $f$ at hand. For $f$ being sufficiently smooth, a valid RHS can be constructed by computing approximate single scale approximations of $\left.f\right|_{\Omega_{i}}$ on some appropriate level using suitable quadrature, and then by applying inverse wavelet transforms.

Remark 3.8. As subblocks of an $s^{*}$-admissible matrix $\mathbf{A}$, both the diagonal blocks $\mathbf{A}^{(i, i)}$ and the off-diagonal blocks $\mathbf{A}^{(i, j)}$ for $i \neq j$ are $s^{*}$-admissible. In a quantitative sense, however, an approximate application of $\mathbf{A}^{(i, j)}$ for $i \neq j$ can be expected to be much more expensive than that of $\mathbf{A}^{(i, i)}$. Indeed, the entries of $\mathbf{A}^{(i, j)}$ correspond to pairs of wavelets that are piecewise smooth with respect to generally uncorrelated partitions, making the problem of approximating them using suitable quadrature much more demanding. In view of this, it can be expected that our approach of before solving on $\Omega_{i}$ deleting for $j \neq i$ all coefficients $\mathbf{u}_{k-1, \lambda}^{(j)}$ for $\lambda \in \nabla^{(j)}$ with $\operatorname{supp} \psi_{\lambda}^{(j)} \cap \Omega \subset \Omega_{i}$ also gives quantitative advantages, since it makes $\mathbf{u}_{k}^{(j)}$ more sparse.

Remark 3.9. To approximately solve the problems on the subdomains, for simplicity we suggested applying some inexact damped Richardson iterations. This is the approach from the second adaptive wavelet method proposed by Cohen, Dahmen and DeVore (CDD02 ). Alternatively, one can apply a few iterations of the first method introduced by these authors (CDD01]; see also [GHS07]). This method is somewhat more difficult to describe, but in practice it leads to quantitatively better results. In our numerical experiments we have applied this method.

\section{Construction of the limits of the SEQuences on the Subdomains}

In this section, we prove Part (a) of Theorem 3.4, i.e., the convergence for each $i \in\{0, \ldots, m-1\}$ of $\left(u_{k}^{(i)}\right)_{k \in i+1+m \mathbb{N}_{0}}$ in $H_{0}^{t}\left(\Omega_{i}\right)$. We give explicit expressions for the limit functions, that in the next section will be shown to be sufficiently smooth to 


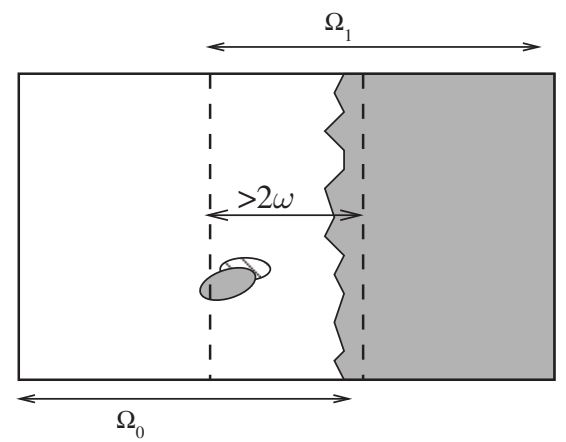

FiguRE 1. $\operatorname{supp} \psi_{\lambda}^{(0)}$ and $\operatorname{supp} \tilde{\psi}_{\lambda}^{(0)}$ for $\lambda \in \nabla^{(0)}$ with $\operatorname{supp} \psi_{\lambda}^{(0)} \not \subset \Omega^{(1)}$, and supp $u_{k}^{(1)}$ for some odd $k$ assuming (3.5).

ensure Theorem 3.4(b). Before giving a formal proof in the general case of having $m$ subdomains, we start with sketching the idea for 2 subdomains.

For $k$ odd, $\mathbf{u}_{k, \mu}^{(1)}$ is defined to be zero when $\operatorname{supp} \psi_{\mu}^{(1)} \cap \Omega \subset \Omega_{0}$. Now from $\operatorname{diam}\left(\operatorname{supp} \psi_{\mu}^{(1)}\right), \operatorname{diam}\left(\operatorname{supp} \psi_{\lambda}^{(0)}\right), \operatorname{diam}\left(\operatorname{supp} \tilde{\psi}_{\lambda}^{(0)}\right) \leq \eta$ and $\operatorname{supp} \psi_{\lambda}^{(0)} \cap \operatorname{supp} \tilde{\psi}_{\lambda}^{(0)} \neq \emptyset$ $\left(\mu \in \nabla^{(1)}, \lambda \in \nabla^{(0)}\right)$, and the assumption (3.5) that $5 \eta \leq \omega$ (already $3 \eta \leq 2 \omega$ suffices), for any $\lambda \in \nabla^{(0)}$ with $\operatorname{supp} \psi_{\lambda}^{(0)} \not \subset \Omega_{1}$,

$$
\mathbf{u}_{k, \lambda}^{(0)}=\tilde{\psi}_{\lambda}^{(0)}\left(u_{k}^{(0)}\right)=\tilde{\psi}_{\lambda}^{(0)}\left(u_{k}-u_{k}^{(1)}\right)=\tilde{\psi}_{\lambda}^{(0)}\left(u_{k}\right) \rightarrow \tilde{\psi}_{\lambda}^{(0)}(u) \quad(k \text { odd } \rightarrow \infty) ;
$$

see Figure 1. For $k$ even, $\mathbf{u}_{k, \lambda}^{(0)}$ is defined to be zero when $\operatorname{supp} \psi_{\lambda}^{(0)} \cap \Omega \subset \Omega_{1}$, and it is equal to $\mathbf{u}_{k-1, \lambda}^{(0)}$ otherwise. From $u_{k}=u_{k}^{(0)}+u_{k}^{(1)} \rightarrow u(k \rightarrow \infty)$, we conclude that

$$
u_{k}^{(1)} \rightarrow u-\sum_{\left\{\lambda \in \nabla^{(0)}: \operatorname{supp} \psi_{\lambda}^{(0)} \not \subset \Omega_{1}\right\}} \tilde{\psi}_{\lambda}^{(0)}(u) \psi_{\lambda}^{(0)} \in H_{0}^{t}\left(\Omega_{1}\right) \quad(k \text { even } \rightarrow \infty) .
$$

To relate this result with the analysis in the general case, let $\phi_{i}$ be a smooth function on $\Omega$, that vanishes outside $\Omega_{i}$, and that is identically 1 on $\Omega_{i}$ except on a sufficiently small strip near $\Omega \backslash \Omega_{i}$. Let $u^{(0,0)}=\phi_{0} u \in H_{0}^{t}\left(\Omega_{0}\right)$ with representation $\left(\mathbf{u}^{(0,0)}\right)^{T} \Psi^{(0)}$. From $\tilde{\psi}_{\lambda}^{(0)}(u)=\mathbf{u}_{\lambda}^{(0,0)}$ when $\operatorname{supp} \psi_{\lambda}^{(0)} \not \subset \Omega_{1}$, we infer that the above limit function from $H_{0}^{t}\left(\Omega_{1}\right)$ can also be written as

$$
\phi_{1}\left(u-u^{(0,0)}\right)+\sum_{\left\{\lambda \in \nabla^{(0)}: \operatorname{supp} \psi_{\lambda}^{(0)} \subset \Omega_{1}\right\}} \mathbf{u}_{\lambda}^{(0,0)} \psi_{\lambda}^{(0)} .
$$

Now in the general case of having $m$ subdomains, let $\left(u_{k}\right)_{k \in \mathbb{N}_{0}}$ be a sequence of approximations to $u$ such that $u_{k}=\sum_{i=0}^{m-1} u_{k}^{(i)}, u_{k}^{(i)}=\left(\mathbf{u}_{k}^{(i)}\right)^{T} \Psi^{(i)}$, and for $k \geq 1$ and $j \neq(k-1) \bmod m$,

$$
\mathbf{u}_{k, \lambda}^{(j)}:=\left\{\begin{array}{cl}
0 & \text { when supp } \psi_{\lambda}^{(j)} \cap \Omega \subset \Omega_{(k-1) \bmod m}, \\
\mathbf{u}_{k-1, \lambda}^{(j)} \text { or } 0 & \text { otherwise. }
\end{array}\right.
$$

An example of such a sequence is given by the one produced by MultSchw. The possibility that $\mathbf{u}_{k, \lambda}^{(j)}$ is set to zero also when $\operatorname{supp} \psi_{\lambda}^{(j)} \cap \Omega \not \subset \Omega_{(k-1) \bmod m}$ allows us to include the effect of a coarsening step. In that case $u_{k}$ should read as the iterand after coarsening. 
Due to the cyclic character of the assumption (4.1), it is sufficient to prove the convergence of the sequence $\left(u_{k}^{(m-1)}\right)_{k \in m \mathbb{N}_{0}}$. Apart from proving convergence, we will derive an expression for the limit.

With $\eta$ from (3.5), for some $\theta \geq-\frac{1}{3}, 0 \leq i \leq m-1$, let $\phi_{i, \theta} \in C_{0}^{\infty}\left(\mathbb{R}^{n}\right)$ with

$$
\operatorname{supp} \phi_{i, \theta} \cap \Omega \subset \Omega_{i}(-(1+3 \theta) \eta), \quad \phi_{i, \theta} \equiv 1 \text { on } \Omega_{i}(-(2+3 \theta) \eta) .
$$

We define $\left(\chi_{i, \theta}\right)_{0 \leq i \leq m-1}$ by $\chi_{0, \theta}=\phi_{0, \theta}$, and for $i>0$ by

$$
\chi_{i, \theta}=\phi_{i, \theta}\left(1-\chi_{i-1, \theta}\right)+\chi_{i-1, \theta} .
$$

\section{Proposition 4.1. It holds that}

(1) $\operatorname{supp} \chi_{i, \theta} \cap \Omega \subset \bigcup_{j=0}^{i} \Omega_{j}(-(1+3 \theta) \eta)$,

(2) $\chi_{i, \theta} \equiv 1$ on $\bigcup_{j=0}^{i} \Omega_{j}(-(2+3 \theta) \eta)$.

Proof. By the definition of $\chi_{i, \theta}$, we have

$$
\begin{aligned}
& \phi_{0, \theta}(x)=1 \vee \cdots \vee \phi_{i, \theta}(x)=1 \Longrightarrow \chi_{i, \theta}(x)=1, \\
& \phi_{0, \theta}(x)=0 \wedge \cdots \wedge \phi_{i, \theta}(x)=0 \Longrightarrow \chi_{i, \theta}(x)=0,
\end{aligned}
$$

from which both statements follow.

Definition 4.2. For $0 \leq i \leq j \leq m-1$, we define $u^{(i, j)} \in H_{0}^{t}\left(\Omega_{i}\right)$ with representation $\left(\mathbf{u}^{(i, j)}\right)^{T} \Psi^{(i)}$ as follows:

$$
\begin{aligned}
& \text { for } j=0, \ldots, m-1 \text { do } \\
& \begin{aligned}
u^{(j, j)} & :=\phi_{j,-\frac{1}{3}}\left(u-\sum_{i=0}^{j-1} u^{(i, j-1)}\right)+\sum_{i=0}^{j-1} \sum_{\left\{\lambda \in \nabla^{(i)}: \operatorname{supp} \psi_{\lambda}^{(i)} \cap \Omega \subset \Omega_{j}\right\}} \mathbf{u}_{\lambda}^{(i, j-1)} \psi_{\lambda}^{(i)} \mathbf{u}_{\lambda}^{(i, j-1)} \psi_{\lambda}^{(i)} \quad(0 \leq i \leq j-1) \\
u^{(i, j)} & :=u^{(i, j-1)}-\sum_{\left\{\lambda \in \nabla^{(i)}: \operatorname{supp} \psi_{\lambda}^{(i)} \cap \Omega \subset \Omega_{j}\right\}}(0 \leq i \leq 1)
\end{aligned}
\end{aligned}
$$

From $\operatorname{diam}\left(\operatorname{supp} \psi_{\lambda}^{(i)}\right) \leq \eta$ and the definition of $u^{(i, j)}$ for $j>i$ or (4.1), one infers the following result.

Proposition 4.3. For $0 \leq i<j \leq m-1, u^{(i, j)}$ vanishes on

$$
\Omega_{i+1}(-\eta) \cup \cdots \cup \Omega_{j}(-\eta) .
$$

For $0 \leq i \leq m-1, u_{k}^{(i)}$ vanishes on

$$
\Omega_{i+1}(-\eta) \cup \cdots \cup \Omega_{(k-1) \bmod m}(-\eta)
$$

when $(k-1) \bmod m>i$, and on

$$
\Omega_{i+1}(-\eta) \cup \cdots \cup \Omega_{m-1}(-\eta) \cup \Omega_{0}(-\eta) \cup \cdots \cup \Omega_{(k-1) \bmod m}(-\eta)
$$

when $(k-1) \bmod m<i$ (and $k \geq m)$.

Proposition 4.4. It holds that $\chi_{j,-\frac{1}{3}} u=\sum_{i=0}^{j} u^{(i, j)}$.

Proof. For $j=0$, we have $\chi_{0,-\frac{1}{3}} u=\phi_{0,-\frac{1}{3}} u=u^{(0,0)}$. Let the statement be valid for $j-1>0$, then we have $\chi_{j,-\frac{1}{3}} u=\phi_{j,-\frac{1}{3}}\left(u-\chi_{j-1,-\frac{1}{3}} u\right)+\chi_{j-1,-\frac{1}{3}} u=$ $\phi_{j,-\frac{1}{3}}\left(u-\sum_{i=0}^{j-1} u^{(i, j-1)}\right)+\sum_{i=0}^{j-1} u^{(i, j-1)}=u^{(j, j)}+\sum_{i=0}^{j} u^{(i, j)}$. 
Lemma 4.5. For $0 \leq j \leq m-1, \ell \in \mathbb{N}$,

$$
\left\|\chi_{j, j}\left(u^{(j, j)}-u_{\ell m+j+1}^{(j)}\right)\right\|_{H^{t}\left(\Omega_{j}\right)} \lesssim \sum_{i=0}^{j}\left\|u-u_{\ell m+i+1}\right\|_{H^{t}(\Omega)} .
$$

Before giving the proof, we note that as a corollary of this lemma and the assumption (3.5) that $\Omega \subset \bigcup_{i=0}^{m-1} \Omega(-(3 m-1) \eta)$ we have the following result.

Theorem 4.6. With $u^{(m-1)}:=u^{(m-1, m-1)}$, for $\ell \in \mathbb{N}$,

$$
\left\|u^{(m-1)}-u_{(\ell+1) m}^{(m-1)}\right\|_{H^{t}\left(\Omega_{m-1}\right)} \lesssim \sum_{i=0}^{m-1}\left\|u-u_{\ell m+i+1}\right\|_{H^{t}(\Omega)} .
$$

Now invoking the bound (3.2) for $\left\|u-u_{\ell m+i+1}\right\|_{H^{t}(\Omega)}$, and realizing that for any $0 \leq i<m-1$ it is at most some constant factor larger than for $i=m-1$ ( $\rho$ and $m$ are absolute constants), we conclude part (a) of Theorem 3.4 for $i=m-1$. The statement for the other $i$ is proven analogously.

Proof of Lemma 4.5. Since $\chi_{0,0}=\phi_{0,0}$, supp $\phi_{0,0} \cap \Omega \subset \Omega_{0}(-\eta)$, for $i \neq 0 u_{\ell m+1}^{(i)}$ vanishes on $\Omega_{0}(-\eta)$, and $\phi_{0,-\frac{1}{3}} \equiv 1$ on $\Omega_{0}(-\eta)$, we have

$$
\chi_{0,0}\left(u^{(0,0)}-u_{\ell m+1}^{(0)}\right)=\phi_{0,0}\left(\phi_{0,-\frac{1}{3}} u-u_{\ell m+1}\right)=\phi_{0,0}\left(u-u_{\ell m+1}\right) .
$$

The statement for $j=0$ now follows by the smoothness of $\phi_{0,0}$.

Let us now consider $j>0$. From $\chi_{j, j}=\chi_{j, j} \chi_{j,-\frac{1}{3}}$, Proposition 4.4, and $\operatorname{supp} \chi_{j, j} \cap \Omega \subset \bigcup_{i=0}^{j} \Omega_{i}(-\eta)$ whereas for $i>j, u_{\ell m+j+1}^{(i)}$ vanishes on that set, we have

$$
\chi_{j, j}\left(u-u_{\ell m+j+1}\right)=\chi_{j, j} \sum_{i=0}^{j} u^{(i, j)}-\chi_{j, j} \sum_{i=0}^{j} u_{\ell m+j+1}^{(i)} .
$$

Since for $i<j, u^{(i, j)}$ and $u_{\ell m+j+1}^{(i)}$ vanish on $\Omega_{j}(-\eta) \cup \Omega_{j-1}(-\eta) \cup \cdots \cup \Omega_{i+1}(-\eta)$, by definition of $\chi_{j, j}$ for those $i$ we have $\chi_{j, j}\left(u_{\ell m+j+1}^{(i)}-u^{(i, j)}\right)=\chi_{i, j}\left(u_{\ell m+j+1}^{(i)}-u^{(i, j)}\right)$, and so

$$
\begin{aligned}
& \chi_{j, j}\left(u^{(j, j)}-u_{\ell m+j+1}^{(j)}\right)=\chi_{j, j}\left(u-u_{\ell m+j+1}\right)+\sum_{i=0}^{j-1} \chi_{i, j}\left(u_{\ell m+j+1}^{(i)}-u^{(i, j)}\right) \\
&=\chi_{j, j}\left(u-u_{\ell m+j+1}\right)+\sum_{i=0}^{j-1} \chi_{i, j} \sum_{\lambda \in \nabla^{(i, j)}}\left(\mathbf{u}_{\ell m+i+1, \lambda}^{(i)}-\mathbf{u}_{\lambda}^{(i, i)}\right) \psi_{\lambda}^{(i)},
\end{aligned}
$$

where $\nabla^{(i, j)}:=\left\{\lambda \in \nabla^{(i)}: \operatorname{supp} \psi_{\lambda}^{(i)} \cap \Omega \not \subset \Omega_{q}, i+1 \leq q \leq j\right\}$ or a subset of that when in (4.1) additional coefficients are set to zero due to coarsening.

We have $\left\|\chi_{j, j}\left(u-u_{\ell m+j+1}\right)\right\|_{H^{t}(\Omega)} \lesssim\left\|u-u_{\ell m+j+1}\right\|_{H^{t}(\Omega)}$ by the smoothness of $\chi_{j, j}$. Since $\operatorname{diam}\left(\operatorname{supp} \psi_{\lambda}^{(i)}\right) \leq \eta, \operatorname{diam}\left(\operatorname{supp} \tilde{\psi}_{\lambda}^{(i)}\right) \leq \eta$, and for $i<j, \chi_{i, i} \equiv 1$ on $B\left(\operatorname{supp} \chi_{i, j} ; 2 \eta\right) \cap \Omega$ by Proposition 4.1] we have

$$
\begin{aligned}
\chi_{i, j}\left(\mathbf{u}_{\ell m+i+1, \lambda}^{(i)}-\mathbf{u}_{\lambda}^{(i, i)}\right) \psi_{\lambda}^{(i)} & =\chi_{i, j} \tilde{\psi}_{\lambda}^{(i)}\left(u_{\ell m+i+1}^{(i)}-u^{(i, i)}\right) \psi_{\lambda}^{(i)} \\
& =\chi_{i, j} \tilde{\psi}_{\lambda}^{(i)}\left(\chi_{i, i}\left(u_{\ell m+i+1}^{(i)}-u^{(i, i)}\right)\right) \psi_{\lambda}^{(i)} .
\end{aligned}
$$


Since $\chi_{i, j}$ is smooth, and $\psi^{(i)}$ is a Riesz basis for $H_{0}^{t}\left(\Omega_{i}\right)$, we arrive at

$$
\begin{aligned}
& \left\|\chi_{i, j} \sum_{\lambda \in \nabla^{(i, j)}}\left(\mathbf{u}_{\ell m+i+1, \lambda}^{(i)}-\mathbf{u}_{\lambda}^{(i, i)}\right) \psi_{\lambda}^{(i)}\right\|_{H^{t}\left(\Omega_{i}\right)} \\
& \lesssim\left\|\sum_{\lambda \in \nabla^{(i, j)}} \tilde{\psi}_{\lambda}^{(i)}\left(\chi_{i, i}\left(u_{\ell m+i+1}^{(i)}-u^{(i, i)}\right)\right) \psi_{\lambda}^{(i)}\right\|_{H^{t}\left(\Omega_{i}\right)} \\
& \lesssim\left[\sum_{\lambda \in \nabla^{(i, j)}}\left|\tilde{\psi}_{\lambda}^{(i)}\left(\chi_{i, i}\left(u_{\ell m+i+1}^{(i)}-u^{(i, i)}\right)\right)\right|^{2}\right]^{\frac{1}{2}} \\
& \leq\left[\sum_{\lambda \in \nabla^{(i)}}\left|\tilde{\psi}_{\lambda}^{(i)}\left(\chi_{i, i}\left(u_{\ell m+i+1}^{(i)}-u^{(i, i)}\right)\right)\right|^{2}\right]^{\frac{1}{2}} \\
& \lesssim\left\|\chi_{i, i}\left(u_{\ell m+i+1}^{(i)}-u^{(i, i)}\right)\right\|_{H^{t}\left(\Omega_{i}\right)} \lesssim \sum_{q=0}^{i}\left\|u-u_{\ell m+q+1}\right\|_{H^{t}(\Omega)}
\end{aligned}
$$

by the induction hypothesis. Since this holds for any $0 \leq i<j$, the proof is completed.

5. Smoothness of the Limits of the SEQUences $\left(u_{k}^{(i)}\right)_{k \in i+1+m \mathbb{N}_{0}}$

In this section, we show that if $u$ has some representation $\tilde{\mathbf{u}}^{T} \Psi$ with $\tilde{\mathbf{u}} \in \ell_{\tau, \infty}(\nabla)$ for some $s \in\left(0, \frac{d-t}{n}\right)$, then for each of the functions $u^{(i, j)}=\left(\mathbf{u}^{(i, j)}\right)^{T} \Psi^{(i)} \in H_{0}^{t}\left(\Omega_{i}\right)$ from Definition 4.2, we have $\left\|\mathbf{u}^{(i, j)}\right\|_{\ell_{\tau, \infty}\left(\nabla^{(i)}\right)} \lesssim\|\tilde{\mathbf{u}}\|_{\ell_{\tau, \infty}(\nabla)}$. Since this result holds true in particular for $u^{(m-1)}=u^{(m-1, m-1)}$, and analogously also for the limit functions on the other subdomains, we conclude the statement of Theorem 3.4(b).

In order to prove the above result, we give a characterization in terms of membership of a non-standard smoothness space of those $v=\mathbf{v}^{T} \Psi^{(i)} \in H_{0}^{t}\left(\Omega_{i}\right)$ for which $\mathbf{v} \in \ell_{\tau, \infty}\left(\nabla^{(i)}\right)$.

In view of the boundary conditions incorporated in the wavelet construction, for $i \in\{0, \ldots, m-1\}, p, q>0, \nu \geq 0$, let $\stackrel{\circ}{B}_{p, q}^{\nu}\left(\Omega_{i}\right)$ denote the closure in the Besov space $B_{p, q}^{\nu}\left(\Omega_{i}\right)$ of the space of smooth functions on $\Omega_{i}$ that vanish at order $t-1$ at $\partial \Omega \cap \partial \Omega_{i}$, and at order $d-2$ at the interior boundary $\partial \Omega_{i} \cap \Omega$. From the wavelet assumptions, in particular concerning their order of polynomial reproduction $d$, and the fact that they are piecewise smooth and globally $C^{d-2}$ where $\frac{d-t}{n} \geq \frac{1}{2}$, it follows that for $s \in\left(0, \frac{d-t}{n}\right), s n+t-\frac{1}{\tau} \notin\{0, \ldots, d-2\}$,

$$
\mathbf{v} \in \ell_{\tau}\left(\nabla^{(i)}\right) \Longleftrightarrow v=\mathbf{v}^{T} \Psi^{(i)} \in \stackrel{\circ}{B}_{\tau, \tau}^{s n+t}\left(\Omega_{i}\right)
$$

with equivalent (quasi-) norms (see [Coh03, §3.10]).

For given $s \in\left(0, \frac{d-t}{n}\right)$, let us select $s_{0}<s<s_{1}$ with for $r=0,1, s_{r} \in\left(0, \frac{d-t}{n}\right)$ and, with $\tau_{r}:=\left(\frac{1}{2}+s_{r}\right)^{-1}, s_{r} n+t-\frac{1}{\tau_{r}} \notin\{0, \ldots, d-2\}$. Then from

$$
\ell_{\tau, \infty}\left(\nabla^{(i)}\right)=\left[\ell_{\tau_{0}}\left(\nabla^{(i)}\right), \ell_{\tau_{1}}\left(\nabla^{(i)}\right)\right]_{\theta, \infty},
$$

where $\frac{1}{\tau}-\frac{1}{\tau_{0}}=\theta\left(\frac{1}{\tau_{1}}-\frac{1}{\tau_{0}}\right)$, and (5.1) it follows that

$$
\mathbf{v} \in \ell_{\tau, \infty}\left(\nabla^{(i)}\right) \Longleftrightarrow v \in \mathcal{B}^{s}\left(\Omega_{i}\right):=\left[\stackrel{\circ}{B}_{\tau_{0}, \tau_{0}}^{s_{0} n+t}\left(\Omega_{i}\right), \stackrel{\circ}{B}_{\tau_{1}, \tau_{1}}^{s_{1} n+t}\left(\Omega_{i}\right)\right]_{\theta, \infty}
$$

with equivalent (quasi-) norms. Note that the interpolation space at the right-hand side is not a Besov space.

For $p, q>0, \nu \geq 0$, let $\stackrel{\circ}{B}_{p, q}^{\nu}(\Omega)$ denote the closure in the Besov space $B_{p, q}^{\nu}(\Omega)$ of the space of smooth functions on $\Omega$ that vanish at order $t-1$ at $\partial \Omega$. For 
$\nu-\frac{1}{p} \leq d-1$, an equivalent definition of $\stackrel{\circ}{B}_{p, q}^{\nu}\left(\Omega_{i}\right)$ is that of the closure in $B_{p, q}^{\nu}\left(\Omega_{i}\right)$ of the space of smooth functions on $\Omega_{i}$ that vanish at order $t-1$ at $\partial \Omega \cap \partial \Omega_{i}$, and whose support intersected with $\Omega$ is contained in $\Omega_{i}$. Since for $s<\frac{d-t}{n}$ and $\frac{d-t}{n} \geq \frac{1}{2}$, it is $s n+t-\frac{1}{\tau}<d-\left(\frac{d-t}{n}+\frac{1}{2}\right) \leq d-1$, we have $\stackrel{\circ}{B}_{\tau, \tau}^{s n+t}\left(\Omega_{i}\right) \hookrightarrow \stackrel{\circ}{B^{s n+t}}(\Omega)$ by means of the zero extension, and so

$$
\mathcal{B}^{s}\left(\Omega_{i}\right) \hookrightarrow \mathcal{B}^{s}(\Omega):=\left[\stackrel{\circ}{B}_{\tau_{0}, \tau_{0}}^{s_{0} n+t}(\Omega), \stackrel{\circ}{B}_{\tau_{1}, \tau_{1}}^{s_{1} n+t}(\Omega)\right]_{\theta, \infty}
$$

On the other hand, for $\phi_{i} \in C_{0}^{\infty}\left(\mathbb{R}^{n}\right)$ with $\operatorname{supp} \phi_{i} \cap \Omega \subset \Omega_{i}$,

$$
v \mapsto \phi_{i} v: \mathcal{B}^{s}(\Omega) \rightarrow \mathcal{B}^{s}\left(\Omega_{i}\right) \text { is bounded. }
$$

So far we can conclude the following. If $u \in H_{0}^{t}(\Omega)$ has a representation $\tilde{\mathbf{u}}^{T} \Psi$, with for some $s \in\left(0, \frac{d-t}{n}\right), \tilde{\mathbf{u}}=\left[\left(\tilde{\mathbf{u}}^{(0)}\right)^{T} \cdots\left(\tilde{\mathbf{u}}^{(m-1)}\right)^{T}\right]^{T} \in \ell_{\tau, \infty}(\nabla)$, then $\left(\tilde{\mathbf{u}}^{(i)}\right)^{T} \Psi^{(i)} \in \mathcal{B}^{s}\left(\Omega_{i}\right)$, and so $u \in \mathcal{B}^{s}(\Omega)$. For $\phi_{i}$ as above, we have $\phi_{i} u \in \mathcal{B}^{s}\left(\Omega_{i}\right)$, and its representation with respect to $\Psi^{(i)}$ is in $\ell_{\tau, \infty}\left(\nabla^{(i)}\right)$, with (quasi-) norm bounded by some multiple of $\|\tilde{\mathbf{u}}\|_{\ell_{\tau, \infty}(\nabla)}$.

Remark 5.1. From (5.2), (5.3), (5.4), and the existence of a smooth partition of unity with respect to the covering $\left\{\Omega_{i}: 0 \leq i \leq m-1\right\}$ of $\Omega$ because of condition (3.4), it follows that for $s \in\left(0, \frac{d-t}{n}\right), u=\tilde{\mathbf{u}}^{T} \Psi$ with $\tilde{\mathbf{u}} \in \ell_{\tau, \infty}(\nabla)$ if and only if $u \in \mathcal{B}^{s}(\Omega)$. In view of Remark [3.1] we note that $B_{\tau, \tau}^{s n+t}(\Omega) \cap H_{0}^{t}(\Omega)=\stackrel{\circ}{B}_{\tau, \tau}^{s n+t}(\Omega)=$ $\left[\stackrel{\circ}{B}_{\tau_{0}, \tau_{0}}^{s_{0} n+t}(\Omega), \stackrel{\circ}{B}_{\tau_{1}, \tau_{1}}^{s_{1} n+t}(\Omega)\right]_{\theta, \tau} \hookrightarrow \mathcal{B}^{s}(\Omega)$.

In view of Definition 4.2, to prove Theorem 3.4(b) it remains to show that if, for some $s \in\left(0, \frac{d-t}{n}\right), \mathbf{v} \in \ell_{\tau, \infty}\left(\nabla^{(j)}\right)$, then $v_{i, j}:=\sum_{\left\{\lambda \in \nabla^{(j)}: \operatorname{supp} \psi_{\lambda}^{(j)} \cap \Omega \subset \Omega_{i}\right\}} \mathbf{v}_{\lambda} \psi_{\lambda}^{(j)} \in$ $\mathcal{B}^{s}\left(\Omega_{i}\right)$ with (quasi-) norm bounded by some multiple of $\|\mathbf{v}\|_{\ell_{\tau, \infty}\left(\nabla^{(j)}\right)}$. Except for some isolated values of $s \in\left(0, \frac{d-t}{n}\right)$, this will be demonstrated below. Since the mapping $\mathbf{v} \mapsto v_{i, j}$ is linear, an interpolation argument then gives the statement for any $s \in\left(0, \frac{d-t}{n}\right)$.

For $\nu-\frac{1}{p}>t-1, \nu-\frac{1}{p} \notin\{0, \ldots, d-2\}$, another equivalent definition of $\stackrel{\circ}{B}_{p, q}^{\nu}\left(\Omega_{i}\right)$ is given by (see Coh03, §3.10])

$$
\begin{aligned}
\left\{v \in B_{p, q}^{\nu}\left(\Omega_{i}\right): \gamma_{\ell} v\right. & =0 \text { on } \partial \Omega \cap \partial \Omega_{i} \text { for } \ell=0, \ldots, t-1, \\
\gamma_{\ell} v & \left.=0 \text { on } \partial \Omega_{i} \cap \Omega \text { for } \ell=0, \ldots, \min \left(d-2,\left\lfloor\nu-\frac{1}{p}\right\rfloor\right)\right\} .
\end{aligned}
$$

Let $s \in\left(0, \frac{d-t}{n}\right)$. Then $s n+t-\frac{1}{\tau}>t-1$. When $s n+t-\frac{1}{\tau} \notin\{0, \ldots, d-2\}$, select $s_{0}<s<s_{1}$, with $s_{r} \in\left(0, \frac{d-t}{n}\right), s_{r} n+t-\frac{1}{\tau_{r}} \notin\{0, \ldots, d-2\}(r=0,1)$, and $\left\lceil s_{0} n+t-\frac{1}{\tau_{0}}\right\rceil=\left\lceil s_{1} n+t-\frac{1}{\tau_{1}}\right\rceil$. The above characterization of $\stackrel{\circ}{B}_{p, q}^{\nu}\left(\Omega_{i}\right)$ now shows that $\stackrel{\circ}{B}_{\tau_{1}, \tau_{1}}^{s_{1} n+t}\left(\Omega_{i}\right)=B_{\tau_{1}, \tau_{1}}^{s_{1} n+t}\left(\Omega_{i}\right) \cap \stackrel{\circ}{B}_{\tau_{0}, \tau_{0}}^{s_{0} n+t}\left(\Omega_{i}\right)$, and so

$$
\begin{aligned}
\mathcal{B}^{s}\left(\Omega_{i}\right)= & {\left[B_{\tau_{0}, \tau_{0}}^{s_{0} n+t}\left(\Omega_{i}\right) \cap \stackrel{\circ}{B_{\tau_{0}}^{s_{0} n+t}}\left(\Omega_{i}\right), B_{\tau_{1}, \tau_{1}}^{s_{1} n+t}\left(\Omega_{i}\right) \cap \stackrel{\circ}{B}_{\tau_{0}, \tau_{0}}^{s_{0} n+t}\left(\Omega_{i}\right)\right]_{\theta, \infty} } \\
= & {\left[B_{\tau_{0}, \tau_{0}}^{s_{0} n+t}\left(\Omega_{i}\right), B_{\tau_{1}, \tau_{1}}^{s_{1} n+t}\left(\Omega_{i}\right)\right]_{\theta, \infty} \cap \stackrel{\circ}{s_{0} n+t} \underset{\tau_{0}, \tau_{0}}{ }\left(\Omega_{i}\right) } \\
= & \left\{v \in\left[B_{\tau_{0}, \tau_{0}}^{s_{0} n+t}\left(\Omega_{i}\right), B_{\tau_{1}, \tau_{1}}^{s_{1} n+t}\left(\Omega_{i}\right)\right]_{\theta, \infty}:\right. \\
& \gamma_{\ell} v=0 \text { on } \partial \Omega \cap \partial \Omega_{i} \text { for } \ell=0, \ldots, t-1, \\
& \left.\gamma_{\ell} v=0 \text { on } \partial \Omega_{i} \cap \Omega \text { for } \ell=0, \ldots, \min \left(d-2,\left\lfloor s n+t-\frac{1}{\tau}\right\rfloor\right)\right\}
\end{aligned}
$$


Now let $\mathbf{v} \in \ell_{\tau, \infty}\left(\nabla^{(j)}\right)$, then $v_{i, j} \in \mathcal{B}^{s}(\Omega)$. Since $\mathcal{B}^{s}(\Omega)$ is continuously embedded in $\left[B_{\tau_{0}, \tau_{0}}^{s_{0} n+t}(\Omega), B_{\tau_{1}, \tau_{1}}^{s_{1} n+t}(\Omega)\right]_{\theta, \infty}$, and the restriction of functions to $\Omega_{i}$ is bounded from $B_{\tau_{i}, \tau_{i}}^{s_{i} n+t}(\Omega)$ to $B_{\tau_{i}, \tau_{i}}^{s_{i} n+t}\left(\Omega_{i}\right)$, we conclude that $v_{i, j} \in\left[B_{\tau_{0}, \tau_{0}}^{s_{0} n+t}\left(\Omega_{i}\right), B_{\tau_{1}, \tau_{1}}^{s_{1} n+t}\left(\Omega_{i}\right)\right]_{\theta, \infty}$ with (quasi-) norm bounded by some multiple of $\|\mathbf{v}\|_{\ell_{\tau, \infty}\left(\nabla^{(j)}\right)}$. The proof of Theorem 3.4 (b) is completed by noting that above trace operators vanish on $v_{i, j}$.

\section{Numerical EXAmples}

In the following, we test the algorithm MultSchw on one- and two-dimensional Poisson problems with homogeneous Dirichlet boundary conditions. We intend to confirm the convergence and optimality of the method in the sense pointed out in Section 3.1. The model problems will be chosen in such a way that the exact solutions exhibit point singularities either induced by the right-hand side (Section 6.1) or solely by the shape of the domain (Section [6.2), so that, as mentioned in Remark [3.1, adaptive schemes qualitatively outperform methods based on uniform discretizations.

6.1. Poisson equation in the unit interval. As a first example we consider the variational solution of the Poisson equation in the unit interval $\Omega=(0,1)$,

$$
-u^{\prime \prime}=f \quad \text { on } \Omega, \quad u(0)=u(1)=0,
$$

i.e., $n=1, t=1$. The right-hand side $f$ is chosen to be the functional defined by $f(v):=4 v\left(\frac{1}{2}\right)+\int_{0}^{1} g(x) v(x) d x$, where $g(x)=-9 \pi^{2} \sin (3 \pi x)-4$. Consequently, the solution is given by

$$
u(x)=-\sin (3 \pi x)+ \begin{cases}2 x^{2}, & x \in\left[0, \frac{1}{2}\right), \\ 2(1-x)^{2}, & x \in\left[\frac{1}{2}, 1\right] .\end{cases}
$$

This function is contained in the Besov space $B_{\tau, \tau}^{s+1}(\Omega)$, for all $s>0, \tau^{-1}=s+\frac{1}{2}$. From Section 5, we therefore know that $u$ has a representation $\tilde{\mathbf{u}}^{T} \Psi$ with for all $s<d-1, \tilde{\mathbf{u}} \in \ell_{\tau, \infty}(\nabla)$. On the other hand, $u$ is only in $H^{\alpha}(\Omega)$ for $\alpha<\frac{3}{2}$, due to the singularity at 0.5 .

We choose the overlapping domain decomposition $\Omega=\Omega_{0} \cup \Omega_{1}=(0,0.7) \cup$ $(0.3,1)$, and, associated to this covering, we construct a wavelet frame by aggregating local wavelet bases on $\Omega_{0}$ and $\Omega_{1}$, respectively. In particular, as building blocks in this simple construction procedure, we use the biorthogonal spline wavelet bases developed in Pri06 lifted to the subdomains. Especially, we use wavelets with primal spline order $d=2,3$ and 4 , having $\tilde{d}=d$ vanishing moments. The primal functions vanish on $\partial \Omega$ at order 1 , whereas at the internal boundaries (here 0.3 or 0.7 ) they vanish at order $d-2$. No boundary conditions are imposed on the dual basis functions, so that all (primal) wavelets have $\tilde{d}$ vanishing moments.

The construction of the primal and dual interval bases is based on a pair of biorthogonal multiresolution analyses $\left\{V_{j}\right\}_{j \geq j_{0}}$ and $\left\{\tilde{V}_{j}\right\}_{j \geq j_{0}}$ with a sufficiently large coarsest scale $j_{0}$. Note that increasing $j_{0}$ decreases the maximal size of the supports of the primal and dual scaling functions and wavelets. For the cases $d=2,3$ and 4 we used $j_{0}=4$, and for $d=3$ additionally we tested $j_{0}=3$.

Figure 2 shows the decay of the $\ell_{2}$-norm of the residuals of the iterands $\mathbf{u}_{k}$ after the application of COARSE vs. their support length and CPU time, respectively. Recall that the $\ell_{2}$-norm of the residuals is equivalent to $\left\|u-u_{k}\right\|$. For the choices $d=2,3,4$ with $j_{0}=4$, the algorithm appears to converge with the optimal rate $d-1$ with respect to the degrees of freedom, whereas for $d=3, j_{0}=3$, the 

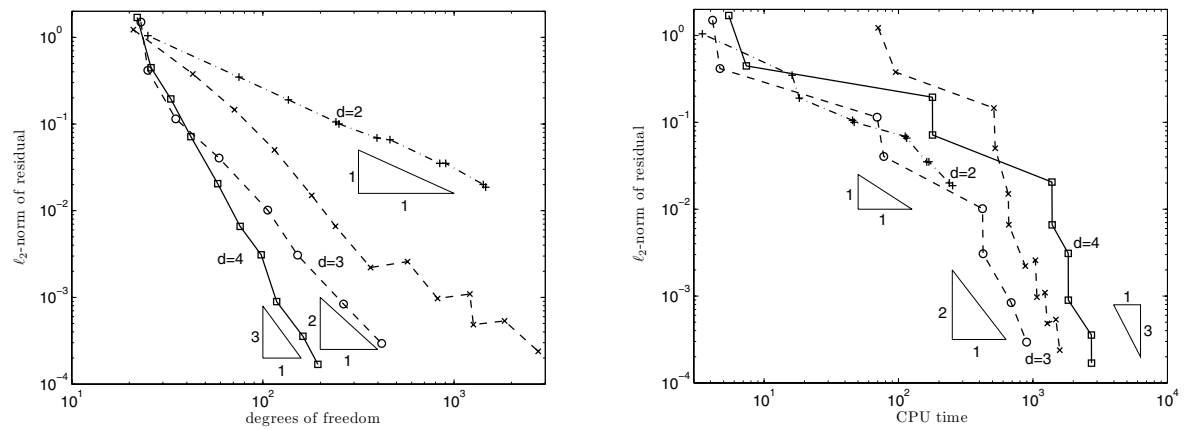

Figure 2. $\ell_{2}$-norms of the residuals of $\mathbf{u}_{k}$ after the application of COARSE versus \#supp $\mathbf{u}_{k}$ (left) and versus CPU time (right). Dashdotted line: $d=2$, circles: $d=3, j_{0}=4$, crosses: $d=3, j_{0}=3$, solid line: $d=4$.

algorithm shows a suboptimal behavior. Concerning CPU times, note that we have implemented a caching strategy for the required entries of the stiffness matrix, which, in some cases, may cause the error, for a number of steps, to decrease faster than the expected optimal convergence rate actually predicts, as it can be observed in the right graphics in Figure 2 .

In the following we focus on the case $d=3$. By checking the maximal diameters of the supports of the wavelets, we conclude that in both cases $j_{0}=4$ and $j_{0}=3$, the sufficient condition (3.5) for the existence of a smooth limit on each subdomain is violated, to a different extent though.

Despite the violation of (3.5), for $d=3, j_{0}=4$, we observe convergence of the iterands on each subdomain. In Figure 3, the distribution of the wavelet coefficients of the final approximation $u_{2 k}$ on each subdomain is depicted. The left picture corresponds to the (final) local approximation $u_{2 k}^{(0)}$ which is plotted in Figure 4 (upper left). Obviously, $u_{2 k}^{(0)}$ is very smooth and consists of a rather equally distributed set of wavelets on small scales. Note that by definition of our modified multiplicative Schwarz scheme, $u_{2 k}^{(0)}$ does not contain contributions from wavelets $\psi_{\lambda}^{(0)}$ whose supports are contained in $\Omega_{1}=(0.3,1)$. The right graphics in Figure 3 and Figure 4 (upper right) address the (final) local approximation $u_{2 k}^{(1)}$. The adaptive scheme plainly detects the singularity at 0.5 where the wavelet coefficients show the characteristic tree-like structure. The additional peaks in the coefficient distribution near the left end of $\Omega_{1}$ can be explained by the fact that the local limit function
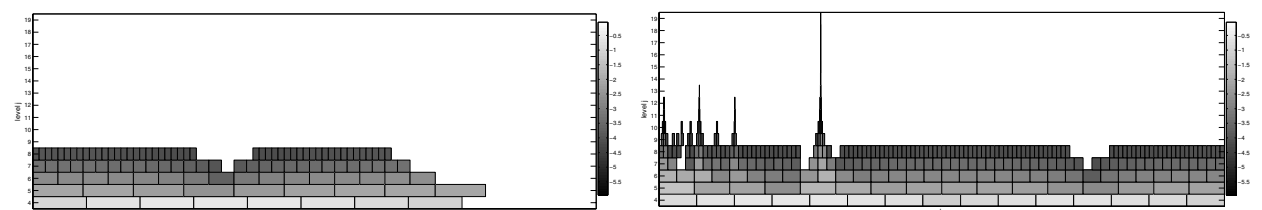

FIGURE 3. Distribution of wavelet coefficients of the final approximation $u_{2 k}$ for $d=3, j_{0}=4$, on $\Omega_{0}$ (left) and on $\Omega_{1}$ (right). 

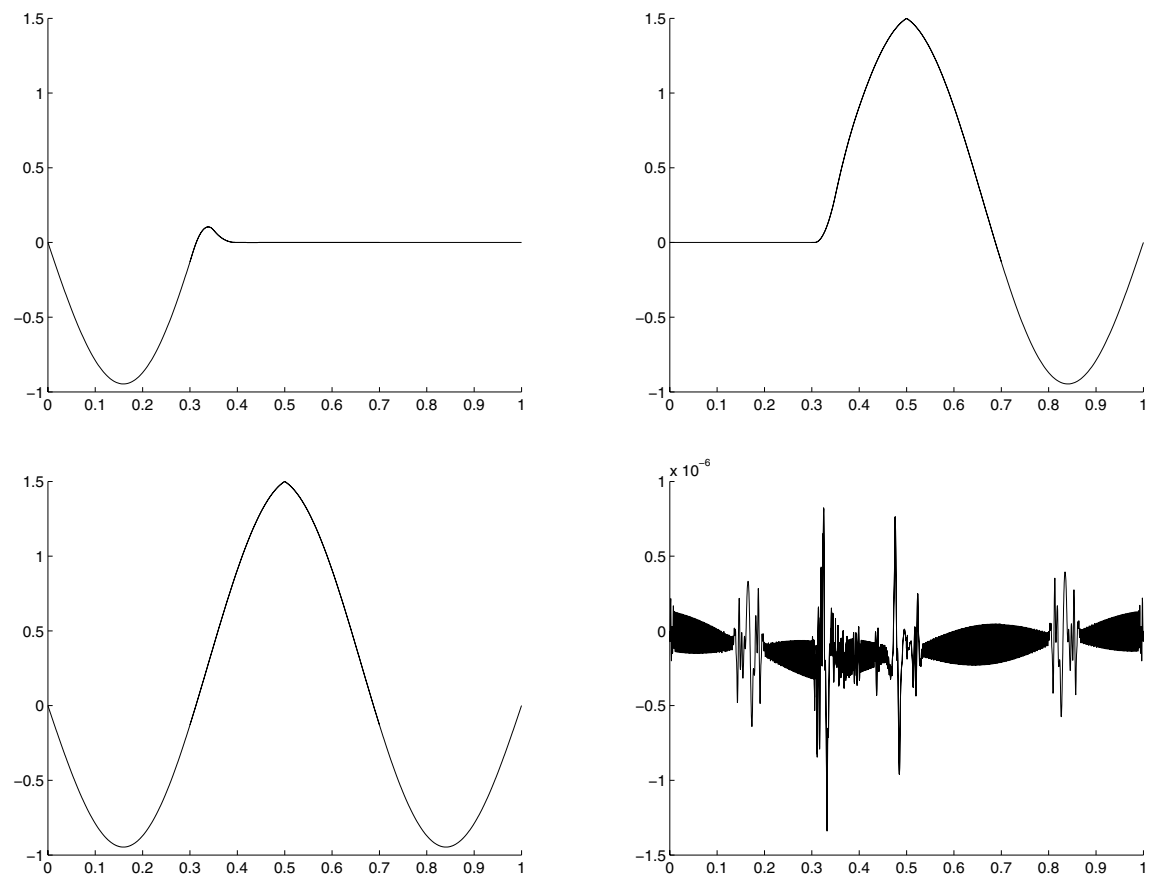

FiguRE 4. Final local approximations $u_{2 k}^{(0)}$ (upper left), $u_{2 k}^{(1)}$ (upper right), produced by MultSchw and the corresponding global approximation $u_{2 k}$ and global pointwise error for $d=3, j_{0}=4$.

$u^{(1)} \in H_{0}^{t}\left(\Omega_{1}\right)$ equals $u-\sum_{\left\{\lambda \in \nabla^{(0)}: \operatorname{supp} \psi_{\lambda}^{(0)} \not \subset \Omega_{1}\right\}} \mathbf{u}_{\lambda}^{(0)} \psi_{\lambda}^{(0)}$, where $u^{(0)}=\left(\mathbf{u}^{(0)}\right)^{T} \Psi^{(0)}$ is the local limit function on $\Omega_{0}$. Since at any fixed point $x \in(0.3,1)$, at most finitely many wavelets in the sum do not vanish, the local adaptive solver detects the natural singularities of these wavelets. The lower pictures in Figure 4 show the global approximation and its corresponding pointwise error. Finally, the computed $\ell_{\tau, \infty}$-norms of the iterands $\mathbf{u}_{2 k}$ for $\tau=\left(\frac{1}{2}+d-1\right)^{-1}$ given in Figure 6 (left) indicate that they are bounded uniformly in $k$.

For $d=3, j_{0}=3$ we observe a different behavior. In particular, in view of Figure 5, one realizes that the local contributions produced by MultSchw for now look completely different and that their amplitudes have an overshoot of about a factor 4 compared to the exact solution. Moreover, in our tests we have observed that they even seem to grow unboundedly. Thus, contrary to the case $j_{0}=4$, no local convergence of the sequences $\left(u_{2 k}^{(i)}\right)_{k \in \mathbb{N}}$ in $H_{0}^{t}\left(\Omega_{i}\right)$ could be observed. In addition, Figure 6 (left) reveals the unbounded growth of the $\ell_{\tau, \infty}$-norms of the iterates in this case, which causes the suboptimal convergence we have observed in Figure 2 (left).

Summarizing, from the different results for $j_{0}=4$ and $j_{0}=3$ we conclude that although condition (3.5) is not strictly necessary for optimality, on the other hand, it should not be violated to a too large extent.

Finally, in this subsection, we compare with two other methods to approximate the solution of the one-dimensional Poisson problem from the span of the frame $\Psi$. 

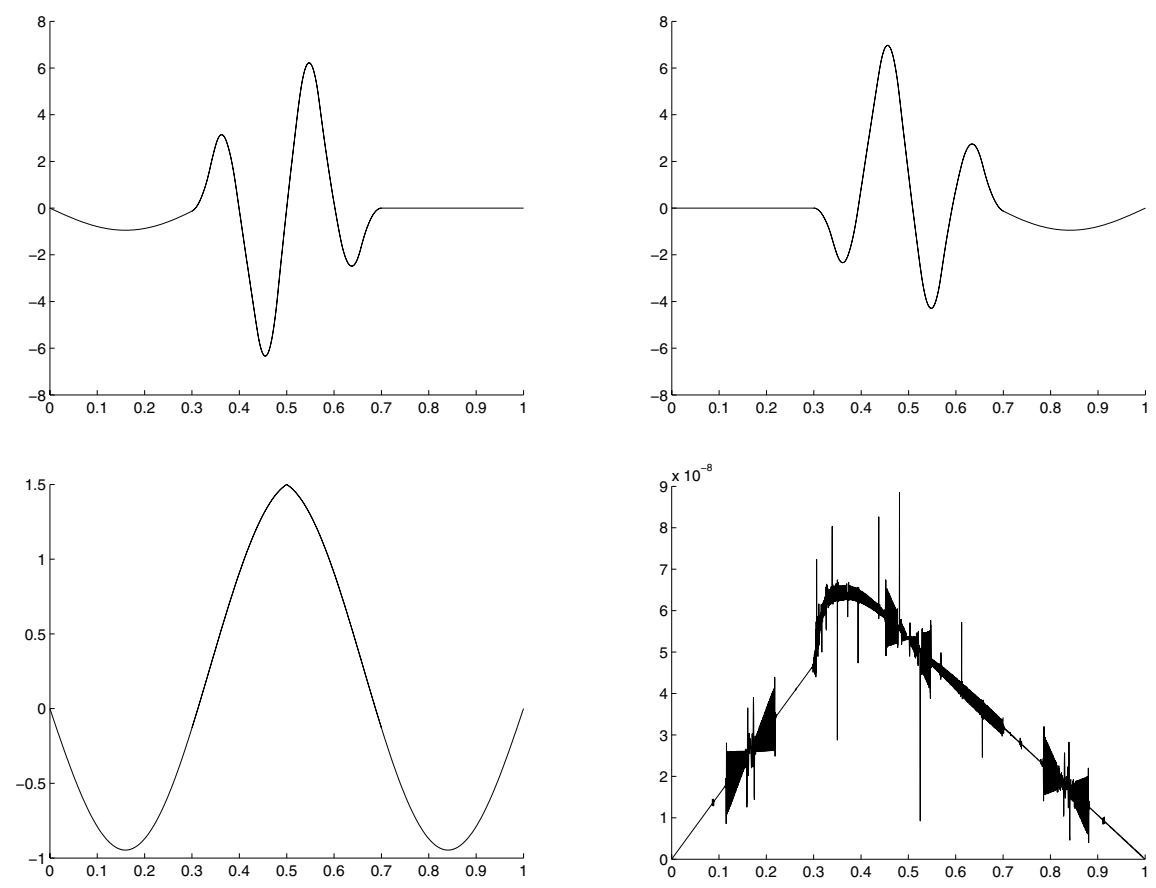

FiguRE 5. Final local approximations $u_{2 k}^{(0)}$ (upper left), $u_{2 k}^{(1)}$ (upper right), produced by MultSchw and the corresponding global approximation $u_{2 k}$ and global pointwise error for $d=3, j_{0}=3$.
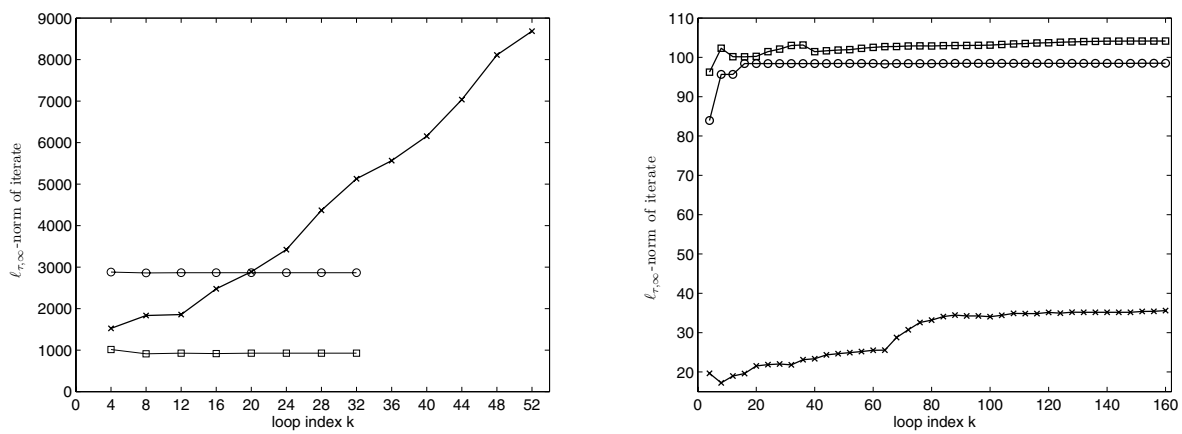

FiguRE 6. Left: $\ell_{\tau, \infty}$-norms of the iterates of MultSchw for the 1D Poisson equation, $d=3, j_{0}=3$ (crosses), $j_{0}=4$ (circles), and for "plain DD", $j_{0}=3$ (boxes), $\tau=2.5^{-1}$. Right: $\ell_{\tau, \infty}$-norms of the iterates of MultSchw for the 2D Poisson equation, $d=3, j_{0}=3$ (crosses), $j_{0}=4$ (circles), and for "plain DD", $j_{0}=4$ (boxes), $\tau=1.5^{-1}$.

We consider the multiplicative Schwarz method without our modification of removing contributions from other subdomains before solving on $\Omega_{i}$ (we will refer 
to this method as "plain DD") and the adaptive steepest descent method developed in $\mathrm{DFR}^{+} 07$. (referred to as "SD"). The latter method is basically an inexact application of the steepest descent method onto the consistent singular system $\mathbf{A u}=\mathbf{f}$. Under a reasonable, but hard to rigorously verify condition on the frame, this method was shown to be of optimal computational complexity. We have used $j_{0}=3$ for "plain DD" and "SD", $j_{0}=4$ for MultSchw, and $d=3$. The results given in Figure 7 show that MultSchw outperforms these two methods. The supports of the iterates and the CPU times needed to attain the same accuracy for MultSchw have been between 5 and 6 times smaller than for "SD". Moreover, MultSchw turns out to be more efficient than "plain DD". For the latter method we observed local convergence as well as bounded $\ell_{\tau, \infty}$-norms of the iterates; cf. Figure 6 (left).
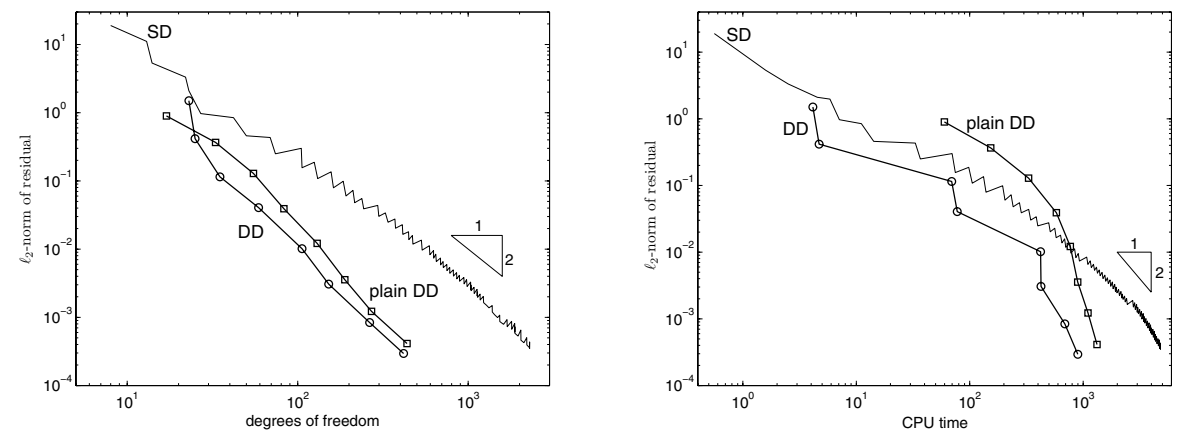

Figure 7. Comparison of MultSchw (DD), an adaptive steepest descent frame method (SD), and MultSchw without extra sparsening (plain DD).

6.2. Poisson equation in the L-shaped domain. We now consider the variational form of Poisson's equation with homogeneous Dirichlet boundary conditions

$$
-\Delta u=f \text { in } \Omega, \quad u_{\mid \partial \Omega}=0
$$

in the L-shaped domain $\Omega=(-1,1)^{2} \backslash[0,1) \times[0,1)$, i.e., $n=2, t=1$. As the exact solution of this model problem we choose the function

$$
\mathcal{S}(r, \theta):=\zeta(r) r^{2 / 3} \sin \left(\frac{2}{3} \theta\right),
$$

with $(r, \theta)$ denoting polar coordinates with respect to the re-entrant corner at the origin, and where $\zeta$ is a smooth function on $[0,1]$ that is identically 1 on $\left[0, r_{0}\right]$ and vanishes on $\left[r_{1}, 1\right]$, for some $0<r_{0}<r_{1}<1$. The function $\mathcal{S}$ together with the corresponding right-hand side $-\Delta \mathcal{S}$ is shown in Figure 8 . It is known that $S \in B_{\tau, \tau}^{2 s+1}(\Omega)$, for all $s>0, \tau^{-1}=\left(s+\frac{1}{2}\right.$ ) (see [Dah99]), but only $S \in H^{\alpha}(\Omega)$, for all $\alpha<5 / 3$, although the right-hand side is arbitrarily smooth and vanishes in a neighborhood of the re-entrant corner; see [Gri85] for details.

We decompose $\Omega$ into two overlapping rectangles $\Omega_{0}=(-1,0) \times(-1,1), \Omega_{1}=$ $(-1,1) \times(-1,0)$. Again the wavelet frames used for the discretization of our model problem are constructed by simply lifting wavelet bases on the unit cube to the subdomains and collecting the resulting local bases into a global system of elements. 
Exact Solution

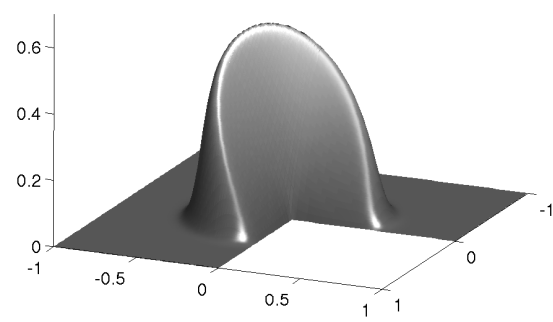

Right-Hand Side

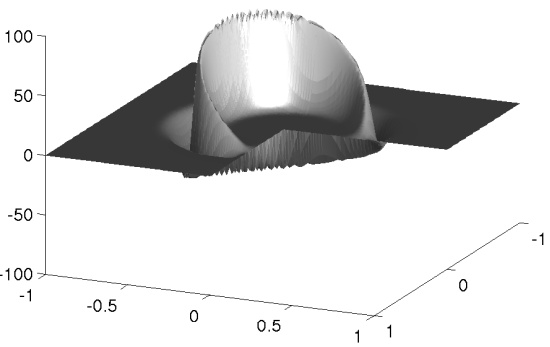

FiguRE 8. Exact solution (left) and right-hand side of the two dimensional test problem.

The reference bases are once again chosen to consist of tensor products of the scaling functions and wavelets constructed in Pri06.

A few comments on the specific choice of the domain decomposition are in order. First, we have to note that assumption (3.4) is not satisfied. Indeed, for any $\omega>0$, $\Omega_{0}(-\omega) \cup \Omega_{1}(-\omega)$ does not cover a part of $\Omega$ near the re-entrant corner. In principle, this can be overcome by adding another polygonal patch $\Omega_{2}$ given by the vertices, say, $(0,0),(0.5,0),(-0.5,-0.5),(0,0.5)$. Nevertheless, for the sake of simplicity, we do not follow this option.

Despite the violation of (3.4), using the homogeneous Dirichlet boundary conditions at the re-entrant corner, still (2.2) can be shown to hold; cf. $\mathrm{DFR}^{+} 07$, Section 4.2]. Consequently, we obtain that the union of the local bases $\bigcup_{i=0}^{m-1} \Psi^{(i)}$ forms a frame for $H_{0}^{t}(\Omega)$, and that the exact multiplicative Schwarz method from Section 2.2 converges. Moreover, in $\left[\mathrm{DFP}^{+} 07\right]$ it has been shown that still $u$ has a representation $\tilde{\mathbf{u}}^{\top} \Psi$ with $\tilde{\mathbf{u}} \in \ell_{\tau, \infty}(\nabla)$ for any $s<\frac{d-1}{2}$. Again, because of the homogeneous Dirichlet boundary conditions at the point where the overlap of the two subdomains gets infinitely small, we have hope that our method is still optimal although the (sufficient) condition (3.5) for optimality is violated. This will be supported by the results given below.

We have tested MultSchw with frames made up of biorthogonal spline wavelet bases of primal and dual order $d=\tilde{d}=2,3,4$, and the choice $j_{0}=4$. For $d=3$ also $j_{0}=3$ has been considered.

Figure 9 shows the convergence histories with respect to the degrees of freedom and computing time. For all setups the optimal convergence rates $\frac{d-1}{2}$ can be observed. Similar to the one-dimensional example from Section 6.1, in case $d=$ 3 , the method performs quantitatively better for $j_{0}=4$ compared to $j_{0}=3$, although now the difference is smaller and also for $j_{0}=3$ optimal convergence can be observed. Moreover, as Figure 10 reveals, again an exceptional oscillatory behavior of the local contributions $u_{2 k}^{(i)}$ on $\Omega_{i}$ to $u_{2 k}$ can be found in the latter case. This phenomenon vanishes for the choice $j_{0}=4$; cf. Figure 10 (upper row). The progression of the $\ell_{\tau, \infty}$-norm of $\mathbf{u}_{2 k}$ during the iteration is investigated in Figure 6 (right), clearly indicating the uniform boundedness for $j_{0}=4$. In case $j_{0}=3$, although generally smaller, the $\ell_{\tau, \infty}$-norms seem to be slowly but constantly increasing. 

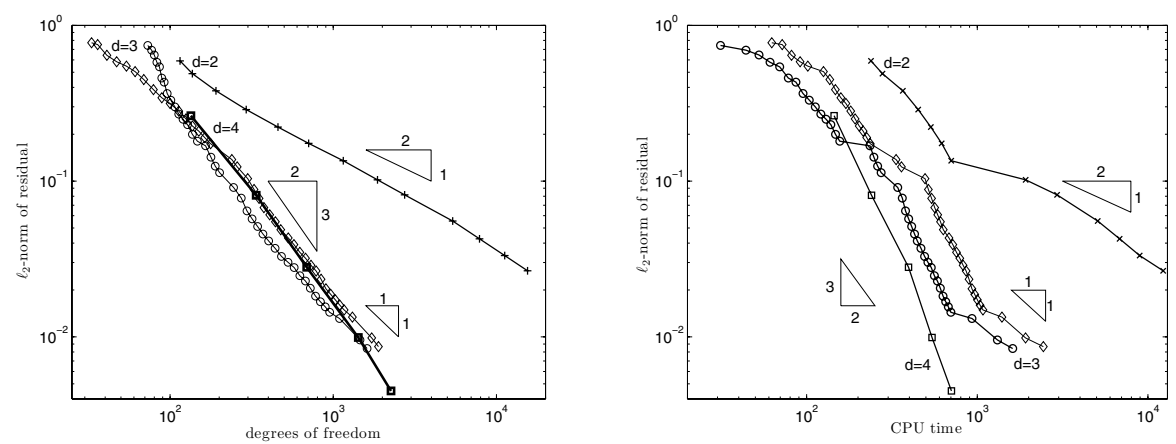

FIGURE $9 . \quad \ell_{2}$-norms of the residuals of $\mathbf{u}_{k}$ after the application of COARSE versus \#supp $\mathbf{u}_{k}$ (left) and versus CPU time (right). Crosses: $d=2$, circles: $d=3, j_{0}=4$, diamonds: $d=3, j_{0}=3$, boxes: $d=4$.
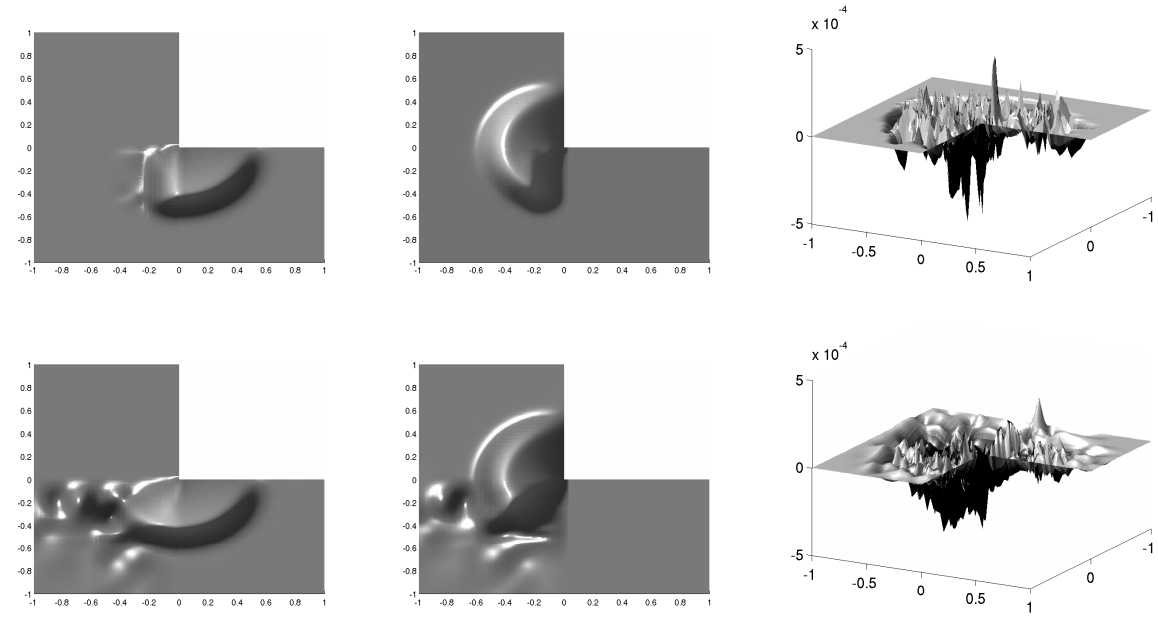

Figure 10. Final local approximations $u_{2 k}^{(0)}$ (left), $u_{2 k}^{(1)}$ (middle) produced by MultSchw and the corresponding global pointwise error (right) for $d=3, j_{0}=4$ (upper row) and $j_{0}=3$ (lower row).

In Figure 11 we once again compare MultSchw with "plain DD" and "SD" for $d=3, j_{0}=4$. In this particular example, we observe that MultSchw and "plain DD" produce approximations being almost equally sparse, but that MultSchw is about a factor 2 faster. The performance of "SD" is significantly worse. In particular, in order to attain the same accuracy, for "SD" about 10 times more degrees of freedom and CPU time were needed than for MultSchw.

Considering Figure 6 (right), one can say that the $\ell_{\tau, \infty}$-norms for "plain DD" are larger than for MultSchw with $j_{0}=3$ and $j_{0}=4$. Moreover, for "plain DD", contrary to MultSchw in combination with $j_{0}=4$, they seem to be very slowly increasing. 

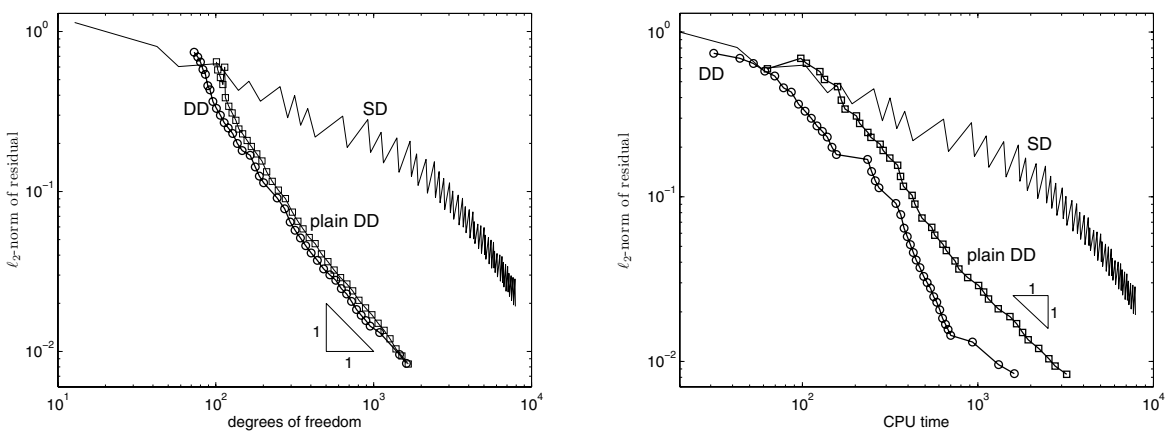

Figure 11. Comparison of MultSchw (DD), an adaptive steepest descent frame method (SD), and MultSchw without extra sparsening (plain DD).

\section{ACKNOWLEDGMENTS}

The authors would like to thank Professor Stephan Dahlke and Dr. Thorsten Raasch (Philipps-University of Marburg) for many helpful discussions and comments on the final version of this manuscript. Parts of the implementation of the methods presented in this paper are based on a code that has been developed in association with Dr. Thorsten Raasch.

\section{REFERENCES}

[Bar05] A. Barinka. Fast Evaluation Tools for Adaptive Wavelet Schemes. Ph.D. thesis, RTWH Aachen, March 2005.

[CDD01] A. Cohen, W. Dahmen, and R. DeVore. Adaptive wavelet methods for elliptic operator equations: Convergence rates. Math. Comp., 70:27-75, 2001. MR.1803124 (2002h:65201)

[CDD02] A. Cohen, W. Dahmen, and R. DeVore. Adaptive wavelet methods II - Beyond the elliptic case. Found. Comput. Math., 2(3):203-245, 2002. MR1907380(2003f:65212)

[CDF92] A. Cohen, I. Daubechies, and J.C. Feauveau. Biorthogonal bases of compactly supported wavelets. Comm. Pur. Appl. Math., 45:485-560, 1992. MR1162365 (93e:42044)

[Coh03] A. Cohen. Numerical Analysis of Wavelet Methods. Elsevier, Amsterdam, 2003. MR 1990555 (2004c:65178)

[Dah99] S. Dahlke. Besov regularity for elliptic boundary value problems in polygonal domains. Appl. Math. Lett., 12(6):31-36, 1999. MR1751404(2001b:35077)

$\left[\mathrm{DFP}^{+} 07\right]$ S. Dahlke, M. Fornasier, M. Primbs, T. Raasch, and M. Werner. Nonlinear and adaptive frame approximation schemes for elliptic PDEs: Theory and numerical experiments. Bericht Nr. 2007-7, Philipps-Universität Marburg, 2007.

[DFR07] S. Dahlke, M. Fornasier, and T. Raasch. Adaptive frame methods for elliptic operator equations. Adv. Comput. Math., 27(1):27-63, 2007. MR2317920(2008c:65366)

$\left[\mathrm{DFR}^{+} 07\right] \quad$ S. Dahlke, M. Fornasier, T. Raasch, R.P. Stevenson, and M. Werner. Adaptive frame methods for elliptic operator equations: The steepest descent approach. IMA J. Numer. Anal., 27(4):717-740, 2007. MR2371829

[DS98] W. Dahmen and R. Schneider. Wavelets with complementary boundary conditionsfunction spaces on the cube. Results Math., 34(3-4):255-293, 1998. MR 1652724 (99h:42057)

[GHS07] T. Gantumur, H. Harbrecht, and R.P. Stevenson. An optimal adaptive wavelet method without coarsening of the iterands. Math. Comp., 77:615-629, 2007. MR 2291830 
[Gri85] P. Grisvard. Elliptic problems in nonsmooth domains, volume 24 of Monographs and Studies in Mathematics. Pitman (Advanced Publishing Program), Boston, MA, 1985. MR.775683 (86m:35044)

[Pri06] M. Primbs. Stabile biorthogonale Spline-Waveletbasen auf dem Intervall. Ph.D. thesis, Universität Duisburg, 2006.

[Sch1890] H.A. Schwarz. Gesammelte Mathematische Abhandlungen, Vol. 2, 133-143. Springer, Berlin, 1890

[Ste03] R.P. Stevenson. Adaptive solution of operator equations using wavelet frames. SIAM J. Numer. Anal., 41(3):1074-1100, 2003. MR.2005196 (2004e:42062)

[SW08] R.P. Stevenson and M. Werner. Computation of differential operators in aggregated wavelet frame coordinates. IMA J. Numer. Anal., 28(2):354-381, 2008.

[Xu92] J. Xu. Iterative methods by space decomposition and subspace correction. SIAM Rev., 34:581-613, 1992. MR:1193013 (93k:65029)

Korteweg-de Vries Institute for Mathematics, University of Amsterdam, Plantage Muidergracht 24, 1018 TV Amsterdam, The Netherlands

E-mail address: rstevens@science.uva.nl

Fachbereich 12 Mathematik und Informatik, Philipps-Universität Marburg, HansMeerwein-Strasse, Lahnberge, D-35032, Marburg, Germany

E-mail address: werner@mathematik. uni-marburg.de 